\title{
Level set Immersed Boundary Method for Coupled Simulation of Air/Water Interaction with Complex Floating Structures
}

\author{
Antoni Calderer ${ }^{\mathrm{a}}$, Seokkoo Kang ${ }^{\mathrm{b}}$, Fotis Sotiropoulos ${ }^{\mathrm{a}, *}$ \\ ${ }^{a}$ St. Anthony Falls Laboratory and Department of Civil Engineering, University of Minnesota, 2 Third Avenue SE, Minneapolis, MN 55414, \\ United States \\ ${ }^{b}$ Civil and Environmental Engineering, Hanyang University, Seoul, South Korea
}

\begin{abstract}
We propose a new computational approach for simulating the coupled interaction between air-water flows and arbitrarily complex floating rigid bodies. The numerical method integrates the fluid-structure interaction (FSI) curvilinear immersed boundary (CURVIB) method of Borazjani et al. (2008) with a level set approach for simulating free surface flows in arbitrarily complex domains. We show that when applying the CURVIB method to simulate two-phase flow FSI problems the approach used to calculate the force imparted on the body is critical for determining the overall accuracy of the method. We develop and demonstrate the accuracy of a new approach for calculating the force, namely the pressure projection boundary condition (PPBC), which is based on projecting the pressure on the surface of the body using the momentum equation along the local normal to the body direction. Extensive numerical tests show that the new approach greatly improves the ability of the method to correctly predict the dynamics of the floating structure motion. To demonstrate the predictive capabilities of the method and its ability to simulate non-linear free surface phenomena, such as breaking waves, we apply it to various two and three-dimensional problems involving complex rigid bodies interacting with a free surface both with prescribed body motion and coupled FSI. We show that for all cases the proposed method yields results in very good accuracy with benchmark numerical data and available experiments. The simulations also reveal the onset of dynamically rich, energetic coherent structures in the air phase induced by the waves generated as the rigid body interacts with the free surface.
\end{abstract}

(C) 2011 Published by Elsevier Ltd.

Keywords: fluid structure-interaction, two-phase free surface flow, large eddy simulation, level set method, immersed boundary method, floating structures, falling wedge

\section{Introduction}

Fluid-structure interaction (FSI) problems involving complex floating structures are encountered in a wide range of engineering applications. Examples include floating oil platforms, wave energy conversion devices, and offshore wind 4 turbines. Numerical simulation of such problems is often the only viable tool for elucidating the underlying physics 5 and optimizing structural designs. In spite of significant computational advances over the last decades, however, the

*Corresponding author.

Email address: fotis@umn.edu (Fotis Sotiropoulos) 
numerical simulation of floating structures of engineering interest continues to pose major challenge. Modeling the coupled interaction of geometrically complex moving structures with a single phase flow field is already a difficult task that requires integrating numerical techniques capable of coupling the fluid and structural domains in a robust and efficient manner. In the case of floating structures, the difficulty of the problem is exacerbated by the inherent two-phase nature of such flows and the non-linear dynamics of the free surface interface dominated by the role of complex phenomena such as turbulence, free surface effects, wave breaking and structure overtopping.

For the most part, previous studies have not considered all the physical aspects required for studying such a complicated problem. Some of these studies treat the problem as a single-phase flow, i.e. instead of solving for the two phases, only the water side is computed while the effect of the air is introduced by satisfying the kinematic and dynamic boundary conditions on the free surface (see for example [1, 2, 3]). Reducing the problem to single-phase flow, and thus neglecting the effect that the air has on the motion of the floating structure, may be a reasonable simplification in specific applications. It is important to consider, however, that important underlying mechanisms of the coupled air/water flow cannot be accounted for by such numerical methods. For example, Iafrati et al. [4] demonstrated that the dissipation of most of the energy induced by a breaking wave occurs via complex vortex structures that develop in the air phase. In addition, in applications such as the study of floating wind turbines being able to resolve the two phases of the flow in a coupled manner is a critical requisite of the modeling approach.

The classic approach for simulating flows around moving bodies is the so-called Arbitrary Lagrangian-Eulerian (ALE), in which the mesh conforms to the moving boundary/interface at all time, and consequently boundary conditions on the interface can be satisfied precisely. Examples of applications are given in [5], for dealing with the solid-fluid interface of a floating structure, and in $[6,7]$ for tracking the free surface interface. The ALE method, however, is not practical for solving situations where the boundary and/or interface is arbitrarily complex, and/or undergoes large deformations. In the particular case that the interface is the free surface the applicability of the ALE approach is limited to problems for which the free surface remains smooth and continuous.

The approach that circumvents the limitations of the ALE methods in such complex situations is by using immersed boundary (IB) methods. IB methods were originally introduced by Peskin [8] to study the blood flow in the heart. In the IB methods the flow equations are solved on a fixed, non-boundary-conforming grid and the effect of the boundary/interface is accounted for by introducing a fictitious force field (see Sotiropoulos and Yang [9] and Mittal and Iaccarino [10] for a review of the different IB methods). A major difficulty of IB methods that is particularly critical in FSI applications arise from the approach used to impose the boundary conditions between the fluid domain, represented by the Eulerian mesh, and the solid body which is typically tracked with a Lagrangian mesh. In the classical IB approach of Peskin the force field is calculated from constitutive laws and imposed on the background grid by employing a discrete delta function. The displacements of the structure follow the motion of the surrounding fluid. In other variations of the IB method such as the hybrid Cartesian/immersed boundary method (HCIB) proposed by Mohd-Yusof [11], the fictitious force added to account for the effect of the interface is not computed explicitly but is introduced implicitly by imposing velocity boundary conditions at the grid nodes located in the vicinity of the 
interface (referred to as IB nodes). To then solve the equations of motion of the moving body, the forces and moments that the fluid exerts to the structure need to be computed by integrating the pressure and shear stresses either directly at the background mesh or at the body surface via extrapolation. In the cut-cell method proposed by Clarke [12] the cells near the boundary are modified so that they conform to its geometry and the velocity boundary condition can be imposed with high accuracy. However, similar to the HCIB, the forces of the fluid acting on the body need to be calculated with special treatments.

The most straightforward technique for computing the forces and moments acting on the structure is by projecting the pressure and shear stresses to all the elements of the Lagrangian mesh of the body and perform a subsequent integration along the surface. Such an approach has been widely applied in single-phase flow problems $[13,14,15]$ as well as in FSI applications with floating structures [1]. In [15] the force at each material point of the structural mesh was calculated by applying inversed distance weight coefficients to the stresses of the nearest IB nodes. Alternatively, Haeri and Shrimpton [16] proposed a method in which two equally spaced auxiliary points located in the wall normal line centered on each material element are defined. In these auxiliary points the stress tensor is reconstructed by interpolating from eight surrounding fluid cells. Finally, the auxiliary points are used to extrapolate with second order accuracy the values of the stress tensor at the center of each Lagrangian element.

The computational cost of the aforementioned projection techniques is considerable and implementation of such algorithms in parallel computing is not straightforward. Several authors have proposed alternative methods to simplify the calculation of the forces and torques. For example, Balaras [17] proposed a method applicable to moving bodies based on the idea of Lai and Peskin [18] in which the stresses are integrated directly at the underlying fluid mesh along a rectangular bounding box. With such an approach there is no need for performing any projection step and the parallelization of the algorithm is straightforward. However, the force calculation expression contains a term that involves integration within the domain inside the bounding box and exterior to the body. This term becomes difficult to compute for geometrically complex bodies and/or moving bodies. Shen et al. [19] extended the method of Balaras to ease the implementation difficulties in complex and moving bodies by splitting the force in two parts, one representing the flow external to the bounding box, and a second representing the virtual flow inside the domain of the body. In the work of Sanders et al. [20] the solid domain is filled with a fictitious fluid that is forced to move at the same velocity as the solid body. The forcing terms to be applied in the equation of motion are obtained by integration within the interior of the solid domain. Borazjani et al. [21] proposed an approach that is also based on integrating the stresses directly at the Eulerian fluid mesh (details of the algorithm are given in [22]). In this case, however, instead of using the surface of a bounding box, the integration is performed along the surface that is constructed by the fluid cells located in the immediate vicinity of the body.

To the best of our knowledge a methodology powerful enough to simulate three-dimensional (3D) FSI problems with two-phase flows based on IB methods does not exist. For example, Sanders et al. [20] presented a two-phase flow formulation employing a fixed grid finite difference IB approach combined with the level set method for tracking the free surface interface. The method was validated by simulating a heave decay test of a cylinder and a roll decay 
test of a rectangular barge, and then applied to simulate a floating buoy. The method, however, has yet to be extended to 3D applications. Similarly, Shen and Chan [23] presented a two-phase flow solver combining the IB method and the VOF. The method was applied only to two-dimensional (2D) problems such as the propagation of waves over a submerged body, or waves induced by a moving bed. The work that is most similar to the numerical method we propose herein is that of Yang et al. [24] who proposed a sharp interface immersed boundary method for carrying out LES of two-phase turbulent flows with a sharp interface level set free surface implementation. The method was applied to simulate the interaction of the free surface interface with bodies undergoing prescribed motion, such as a water entry/exit of a body, landslide induced waves, or the hydrodynamics of a ship. This work however has not been extended to FSI problems.

The objective of this paper is to present a novel and robust numerical method, which integrates the level set formulation with the FSI curvilinear immersed boundary method of Borazjani et al. [21] and is capable of simulating turbulent two-phase free surface flows involving FSI of arbitrarily complex floating structures. However, a major problem arises when extending the FSI algorithm of [21] to floating structures from the approach used to impose the boundary conditions at the boundary of structural domain. To mitigate this difficulty, which yields incorrect dynamics of the structure motion, we develop and assess the accuracy of a new approach for calculating the forces on the body, based on a boundary condition for calculating the pressure on the body using the normal momentum equation. In this paper we present the key elements of the numerical methodology along with results from the application of the method to simulate FSI problems involving geometrically complex structures interacting with a free surface and giving rise to complex phenomena, such as wave breaking and structure overtopping.

The organization of the present paper is as follows: In section 2, the governing equations are presented. In section 3 the numerical methods for solving the coupled flow and structural equations are described, and in section 4 the models are applied to simulate several validation cases.

\section{Governing equations}

\subsection{Navier-Stokes Equations}

We solve the spatially-filtered Navier-Stokes equations governing incompressible flows of two immiscible fluids in non-orthogonal generalized curvilinear coordinates. We adopt the two-fluid, level set formulation, where a single equation is used in all the computational domain taking the corresponding fluid properties values in each fluid phase.

Using compact Newton notation, where repeated indices imply summation, the equations read as follows $(i, j, k, l=$ $1,2,3)$ :

$$
J \frac{\partial U^{i}}{\partial \xi^{i}}=0
$$

$$
\frac{1}{J} \frac{\partial U^{j}}{\partial t}=\frac{\xi_{l}^{i}}{J}\left(-\frac{\partial}{\partial \xi_{j}}\left(U^{j} u_{l}\right)+\frac{1}{\rho(\phi) R e} \frac{\partial}{\partial \xi^{j}}\left(\mu(\phi) \frac{\xi_{l}^{j} \xi_{l}^{k}}{J} \frac{\partial u_{l}}{\partial \xi^{k}}\right)-\right.
$$




$$
\left.-\frac{1}{\rho(\phi)} \frac{\partial}{\partial \xi^{j}}\left(\frac{\xi_{l}^{j} p}{J}\right)-\frac{1}{\rho(\phi)} \frac{\partial \tau_{l j}}{\partial \xi^{j}}-\frac{\kappa}{\rho(\phi) W e^{2}} \frac{\partial h(\phi)}{\partial x_{j}}+\frac{\delta_{i 2}}{F r^{2}}\right),
$$

In the above equations: $\phi$ is the level set function (see below for details), $\xi^{i}$ are the curvilinear components, $\xi_{l}^{i}$ are the transformation metrics, $J$ is the Jacobian of the transformation, $U^{i}$ are the contravariant volume fluxes, $u_{i}$ are the Cartesian velocity components, $\rho$ is the density, $\mu$ is the dynamic viscosity, $p$ is the pressure, $\tau_{l j}$ is the sub-grid stress (SGS) tensor, $\kappa$ is the curvature of the interface, $\delta_{i j}$ is the Kronecker delta, $h$ is the smoothed Heaviside function, and $R e, F r$, and $W e$ are the dimensionless Reynolds, Froude, and Weber numbers, respectively, defined as follows:

$$
R e=\frac{U L \rho_{\text {water }}}{\mu_{\text {water }}}, F r=\frac{U}{\sqrt{g L}}, W e=U \sqrt{\frac{\rho_{\text {water }} L}{\sigma}}
$$

where, $U$ and $L$ are the characteristic velocity and linear dimension, $\rho_{\text {water }}$ and $\mu_{\text {water }}$, are the density and dynamic viscosity of the water phase, $g$ is the gravity, and $\sigma$ is the surface tension. The subgrid scale stress tensor $\tau_{l j}$ in Eq. (2) is modeled in the present work as described in [25] using the dynamic Smagorinsky eddy viscosity model of [26].

In the level set method, the interface is tracked using the signed distance function $\phi(x, t)$, also known as the level set function, which is an scalar function defined in the whole computational domain, measuring the minimum distance from any point $x$ in the fluid to the closest point of the free surface interface. The interface is located at the level $\phi=0$, and the sign is positive in the liquid phase, and negative in the gas phase.

The jump condition of the density and viscosity fields at the interface in a level set approach is taken to be continuous, and is smeared over a thin layer of thickness $2 \epsilon$ to prevent the formation of numerical instabilities. It can be expressed as follows:

$$
\begin{aligned}
& \rho(\phi)=\rho_{\text {air }}+\left(\rho_{\text {water }}-\rho_{\text {air }}\right) h(\phi), \\
& \mu(\phi)=\mu_{\text {air }}+\left(\mu_{\text {water }}-\mu_{\text {air }}\right) h(\phi),
\end{aligned}
$$

where the smoothed Heaviside function [27] $h(\phi)$ is

$$
h(\phi)= \begin{cases}0 & \phi<-\epsilon, \\ \frac{1}{2}+\frac{\phi}{2 \epsilon}+\frac{1}{2 \pi} \sin \left(\frac{\pi \phi}{\epsilon}\right) & -\epsilon \leq \phi \leq \epsilon, \\ 1 & \epsilon<\phi,\end{cases}
$$

Typical values for $\epsilon$ are between one and three times the length of the smallest grid cell. Our experience shows, however, that for problems in which the interface undergoes rapid deformations due to complex phenomena such us air/water entrainments or wave breaking, it is necessary to employ larger values of $\epsilon$ that can be up to the length of six or eight grid cells for the most extreme scenarios. The need for an increased number of grid cells within the transition region has been investigated and discussed in [28, 29]. The study of Iafrati and Campana[28] shows that spurious velocity effects are introduced in the interior of the transition layer caused by the smearing of the interface. If the thickness $\epsilon$ of the layer is sufficiently large the spurious effects remain confined within the transition layer without altering the exterior flow. It was also shown that larger interface thickness is required for increased Reynolds number flows. The kinematic and dynamic boundary conditions ensuring continuity of the velocity and the normal and tangential stresses at the interface, are intrinsic in the current formulation and are satisfied in a smooth manner. 


\subsection{Level set equations}

The motion of the free surface interface can be modeled by the level set method proposed by Osher and Sethian [30]. The spatially filtered advection equations in generalized curvilinear grids will assume the form:

$$
\frac{1}{J} \frac{\partial \phi}{\partial t}+U^{j} \frac{\partial \phi}{\partial \xi^{j}}=-\tau_{i j}^{L}
$$

where $\tau_{i j}^{L}$ is the sub-grid scale stress tensor responsible of the effect of the unresolved subgrid scales on the level set field. In the present model the effect of $\tau_{i j}^{L}$ is neglected assuming that the residual field of $\phi$ is small and its overall contribution to the energy containing scales is negligible.

As Eqn. (7) is integrated in time to determine $\phi$, there is no guarantee that the resulting solution will satisfy the required, for a distance function, unit gradient condition $|\nabla \phi|=1$. Such incosistent solution will in turn lead to poor conservation of mass between the two fluids. This problem is circumvented by solving the following mass conserving re-initialization equation proposed by Sussman and Fatemi [31]:

$$
\frac{\partial \phi}{\partial \tau}+S\left(\phi_{0}\right)(|\nabla \phi|-1)=\lambda \tilde{\delta}(\phi)|\nabla \phi|
$$

where $\tau$ denotes a pseudo-time, $\phi_{0}$ the distance function at the initial step of the pseudo-time iteration procedure, $S\left(\phi_{0}\right)$ is the smoothed sign function, $\tilde{\delta}(\phi)$ is the smoothed delta function, and $\lambda$ a cell dependent coefficient. A detailed description of the method in the context of curvilinear coordinates can be found in Kang and Sotiropoulos [32].

\subsection{Equations of motion for rigid bodies}

For computing the general 6 degrees of freedom (DOF) motion of 3D rigid bodies we use the Lagrangian form of Newton's second law, i.e. the linear and angular momentum equations. With no loss of generality we write the equations for a single body, although the formulation can be extended to multiple bodies, expressed along the principle axes. Under the above assumptions, the general form of the equations for a body mounted on an elastic and damped system can be written in the inertial frame of reference in the following form ( $i=1,2,3)$ :

$$
M \frac{\partial^{2} X^{i}}{\partial t^{2}}+C \frac{\partial X^{i}}{\partial t}+K X^{i}=F_{\text {fluid }}^{i}+F_{e x t}^{i}
$$

$$
I \frac{\partial^{2} \Theta^{i}}{\partial t^{2}}+C \frac{\partial \Theta^{i}}{\partial t}+K \Theta^{i}=M_{\text {fluid }}^{i}+M_{e x t}^{i}
$$

where Eq. (9) represents the pure translation motion, and $X_{i}$ are the components of the position vector, $M$ is the mass of the structure, $C$ the damping coefficient, $K$ the spring stiffness coefficient, $F_{\text {fluid }}^{i}$ the components of the force exerted by the fluid, and $F_{e x t}^{i}$ the external forces. The case of pure rotation is represented by Eq. (10), and $\Theta^{i}$ denote the components of the relative angle of rotation vector, $I$ the moment of inertia, and $M_{\text {fluid }}^{i}$ and $M_{\text {ext }}^{i}$ the moments in respect to the axis of rotation exerted respectively by the fluid, and external forces. For 6 DOF motions the two sets of the equations, linear and angular, have to be solved concurrently. 
The solid and fluid domains are coupled together via the Dirichlet boundary condition that the fluid velocity field must satisfy on the surface $\Gamma$ of the body, as follows:

$$
u_{i}=\frac{\partial X^{i}}{\partial t}+\varepsilon_{i j k} r_{j} \frac{\partial \Theta^{k}}{\partial t}
$$

The system of second order ordinary differential equations (9) and (10) that govern the motion of the structure is first transformed into a system of first order ordinary differential equations and then integrated in time (see [21] for details).

\section{Numerical Methods}

In this section we outline the numerical methodologies for solving the governing equations in hybrid staggered/nonstaggered curvilinear grids using the fractional step method of Ge and Sotiropoulos [33] as extended to multi-phase flows by Kang and Sotiopoulos [32].

\subsection{Solution of Navier-Stokes equations}

We employ the fractional step method developed by Ge and Sotiropoulos [33], which is briefly described herein. The momentum equations (2) are discretized in space and time with a second-order central differencing scheme for the pressure gradient and viscous and SGS stresses, second-order central differencing or a third-order WENO scheme [34] for the advective terms, and the second-order Crank-Nicholson method for time advancement as follows:

$$
\frac{1}{J} \frac{\mathbf{U}^{*}-\mathbf{U}^{\mathbf{n}}}{\Delta t}=\mathbf{P}\left(p^{n}, \phi^{n}\right)+\frac{1}{2}\left(\mathbf{F}\left(\mathbf{U}^{*}, \mathbf{u}^{*}, \phi^{n+1}\right)+\left(\mathbf{F}\left(\mathbf{U}^{\mathbf{n}}, \mathbf{u}^{\mathbf{n}}, \phi^{n}\right)\right),\right.
$$

where $\mathrm{n}$ indicates the previous time step, $\Delta t$ the time step size, $\mathbf{F}$ is the discrete right hand side of Eq.(2) excluding the pressure term, and $\mathbf{P}$ the discrete pressure gradient term. The continuity condition is discretized with three-point central differencing and is enforced in the second stage of the fractional step method with the following pressure Poisson equation, which is discretized with a second-order central differencing scheme:

$$
-J \frac{\partial}{\partial \xi^{i}}\left(\frac{1}{\rho(\phi)} \frac{\xi_{l}^{i}}{J} \frac{\partial}{\partial \xi^{j}}\left(\frac{\xi_{l}^{j} \Pi}{J}\right)\right)=\frac{1}{\Delta t} J \frac{\partial U^{j, *}}{\partial \xi^{j}},
$$

where $\Pi$ denotes the pressure correction. The pressure and the velocity fields resulting from the first step of the method, can then be updated as follows:

$$
p^{n+1}=p^{n}+\Pi,
$$

$$
U^{i, n+1}=U^{i, *}-J \Delta t \frac{1}{\rho(\phi)} \frac{\xi_{l}^{i}}{J} \frac{\partial}{\partial \xi^{j}}\left(\frac{\xi_{l}^{j} \Pi}{J}\right),
$$

1 The $[25,32]$. 


\subsection{Solution of level set equations}

As detailed in [32] the level set equation is discretized with a third-order WENO scheme [34] in space, and an explicit second-order Runge-Kutta scheme in time. The reinitialization of the level set function is a key aspect of the method to ensure the conservation of mass, and consequently the overall accuracy of the method. As it was proven in [31] accuracy is maximized when the complete area covered by the thickness $\epsilon$ of the interface strip is fully redistanced, and this is achieved when the total re-distancing time $\tau$ is equal to $\epsilon$. The reinitialization time is computed in the following manner:

$$
\tau=n_{\tau} \cdot \Delta \tau,
$$

where $\Delta \tau$ is the time step size, and $n_{\tau}$ is the number of time steps. We have observed that when $\Delta \tau$ is large ( $\Delta \tau>0.1 \Delta x$ ), numerical instabilities arise preventing the method from converging. This is particularly important for problems with complex free surface flow phenomena and will be revisited again in the results section of this paper.

\subsection{Solution of the rigid body equation of motion}

The FSI algorithm adopted for solving the equations of motion is the method of Borazjani and Sotiropoulos [21] and its extension to include free surface flows is described in the next sub-sections.

\subsubsection{Computation of the body forces}

The forces $F_{\text {fluid }}^{i}$ and moments $M_{\text {fluid }}^{i}$ that the fluid exerts to the rigid body and appear in the right hand side of the structural equations of motion (eqns. 9 and 10) are computed by integrating the pressure and the viscous stresses along the surface $\Gamma$ of the body as follows:

$$
F_{\text {fluid }}^{i}=\overbrace{\int_{\Gamma}-p n_{i} d \Gamma}^{F_{f, p}^{i}}+\overbrace{\int_{\Gamma} \tau_{i j} n_{j} d \Gamma}^{F_{f, s}^{i}}
$$

$$
M_{\text {fluid }}^{i}=\overbrace{\int_{\Gamma}-\varepsilon_{i j k} r_{j} p n_{k} d \Gamma}^{M_{f, p}^{i}}+\overbrace{\int_{\Gamma} \varepsilon_{i j k} r_{j} \tau_{k l} n_{l} d \Gamma}^{M_{f, s}^{i}}
$$

where $p$ denotes the pressure, $\tau$ the viscous stress, $r$ the position vector, and $n$ the normal vector. The subscripts $p$ and $s$ in the terms in right hand side of the above equations identify contributions from the pressure and shear forces or moments.

For IB methods in general the computation of the forces and moments in equations (17) and (18) cannot be directly performed. The mesh of the fluid domain does not conform with the structure, hence, the pressure and velocity gradients are not known on the surface of the body. Particularly, in the CURVIB method the nodes of the background curvilinear grid are classified in three categories depending on their position with respect to the immersed body: structural nodes, IB nodes, and fluid nodes (see figure 1). The structural nodes are located within the interior of the immersed body and are excluded from the computational domain. The rest of the nodes are either IB nodes, 


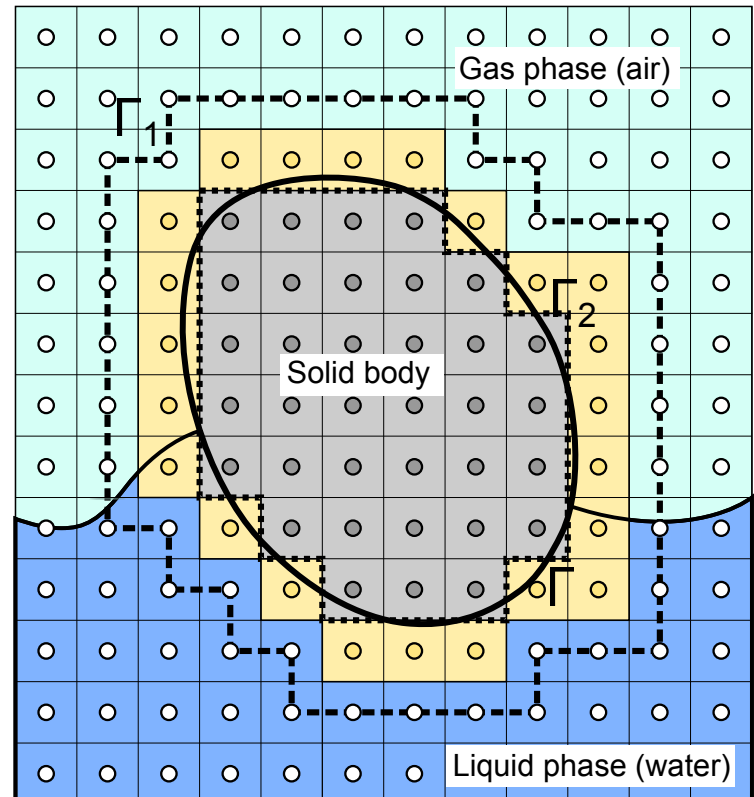

Fluid nodes

Real body surface $\Gamma$

- - - Surface $\Gamma_{1}$ employed in Borazjani et al. [21]

..... Surface $\Gamma_{2}$ employed in the present PPBC method

- Solid phase nodes

- Immersed boundary (IB) nodes

Figure 1: Schematic representation of the node classification in the CURVIB method. The surfaces $\Gamma, \Gamma_{1}$, and $\Gamma_{2}$ are the actual surface of the body and various approximate surfaces on which the pressure field can be integrated to calculate the pressure force acting on the body.

Let $\Gamma$ be the actual surface of the body and $\Gamma_{1}$ the aforementioned approximate surface surrounding the solid body. Let $m_{I B}$ be the mass of the fluid delimited by $\Gamma$ and $\Gamma_{1}$. While in previous studies for single phase problems this mass was neglected, it can be important when the equations are formulated for two-phase flow and include a gravitational force. For instance, let us assume that we have a body submerged in stagnant water. From Archimedes's principle we can easily see that if we integrate the forces along a surface larger than the actual body surface, such as $\Gamma_{1}$, the resulting buoyant force will be higher than the real force felt by the body. Hence, if the method from [21] is applied directly for applications involving gravity, the vertical force will be over-predicted. To overcome this situation we propose an alternative approach referred to as the pressure projection boundary condition (PPBC) method in which the resultant force and moment due to pressure is computed by integrating the pressure distribution on a surface $\Gamma_{2}$. This new approach is presented in the following subsection. 

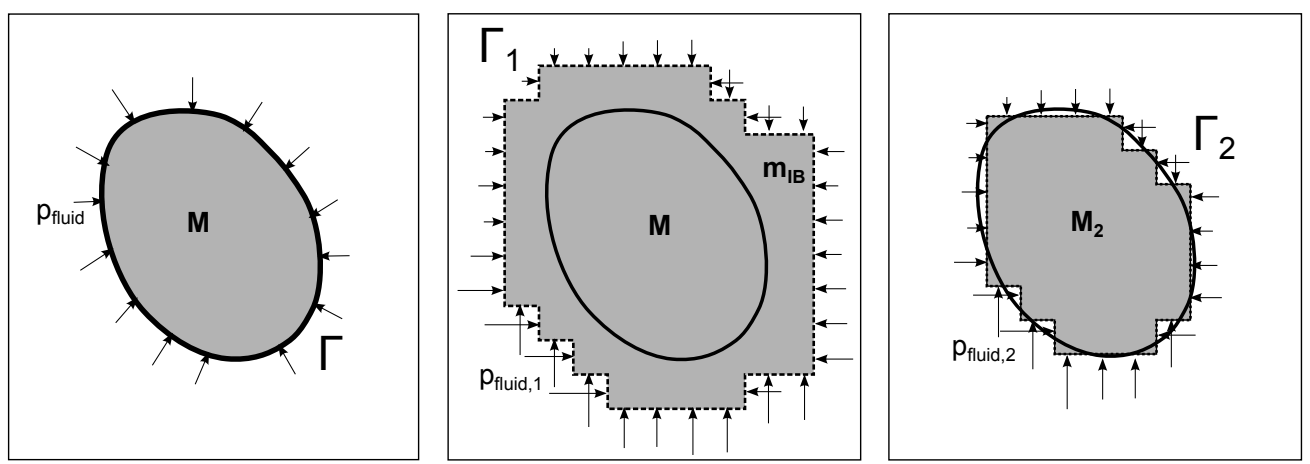

Figure 2: Schematic representation of the various approaches for calculating the pressure force by integrating the pressure field on: 1) the actual body surface $\Gamma$; 2) the surface $\Gamma_{1}$ outlining the volume defined by the IB cells, which was employed in Borazjani et al. [21]; and 3) the approximate surface $\Gamma_{2}$ employed in the proposed PPBC approach. $M$ is the exact or approximate $\left(M_{2}\right)$ mass of the body for each case.

\subsubsection{The pressure projection boundary condition method}

In the proposed PPBC approach, the part of the force $F_{\text {fluid }}$ that is due to pressure is computed by directly integrating the pressure on the surface $\Gamma_{2}$ (see figures 1 and 2). To enable such integration, however, the pressure on $\Gamma_{2}$ has to be appropriately projected from the fluid nodes of the background grid where it is known. We propose a two-step approach for performing this pressure projection. First the pressure is projected to the center of the IB cells which are adjacent to the body. In a second step, the pressure at the center of a given IB cell is projected to its lateral faces belonging in $\Gamma_{2}$ as illustrated in the schematic shown in figure 3 and described in detail as follows.

To obtain the pressure at the IB nodes in the first step, the momentum equation (2) is projected along the direction of the wall normal as done in [13] and applied on the surface of the body. Neglecting viscous and subgrid-scale stresses, the normal momentum equation applied on the body reads as follows:

$$
-\frac{d p}{d n}=\rho(\phi) n^{i}\left(\frac{D u_{i}}{D t}-\frac{\delta_{i 2}}{F r^{2}}\right) \text { on } \Gamma
$$

where $n^{i}$ denotes the unit vector normal to the body surface and $u_{i}$ the velocity components of the body computed with Eq. (11). With reference to figure 3, and since the value of the pressure $p_{c}$ can be readily obtained by interpolating the pressure values between neighboring fluid cells [13], we can obtain the pressure at the IB node $b$ as follows:

$$
p_{b}=p_{c}-d_{c b} \rho_{a} n^{i}\left(\frac{u_{i}^{n}-u_{i}^{n-1}}{\Delta t}-\frac{\delta_{i 2}}{F r^{2}}\right)
$$

where $d_{c b}$ is the distance from points $c$ to $b$, and the superscripts $n$ and $n-1$ denote the current, and previous time steps. The density value $\rho_{a}$ on $\Gamma$ is unknown. However, it can be set to be equal to the density $\rho_{b}$ as the Neumann boundary condition is applied for the distance function $\phi$ normal to the wall. The above equation (20) has been obtained by combining the following two expressions, which are approximations of equation (19) applied on the surface of the body:

$$
-\left(\frac{p_{a}-p_{c}}{d_{c a}}\right)=\rho_{a} n_{a}^{i}\left(\frac{u_{i}^{n}-u_{i}^{n-1}}{\Delta t}-\frac{\delta_{i 2}}{F r^{2}}\right)
$$




$$
-\left(\frac{p_{a}-p_{b}}{d_{b a}}\right)=\rho_{a} n_{a}^{i}\left(\frac{u_{i}^{n}-u_{i}^{n-1}}{\Delta t}-\frac{\delta_{i 2}}{F r^{2}}\right)
$$
where $N_{\text {faces }}$ is the total number of cell faces forming $\Gamma_{2}, S_{a^{\prime}}^{j}$ is the area of the $\mathrm{j}$-th cell face $a^{\prime}$.

$$
F_{f, p, 2}^{i}=\sum_{j=1}^{N_{\text {faces }}} p_{a^{\prime}}^{j} S_{a^{\prime}}^{j} n_{a^{\prime}, i}^{j}
$$

$$
M_{f, p, 2}^{i}=\sum_{l=1}^{N_{\text {faces }}} \varepsilon_{i j k} r_{j}^{l} p_{a^{\prime}}^{l} n_{a^{\prime}, k}^{l} S_{a^{\prime}}^{l}
$$

where $n_{a^{\prime}}$ is taken as the unit normal to the corresponding cell face. A similar expression is used to obtain the pressure at $a^{\prime \prime}$. Once the pressure has been computed at the center of all the faces forming $\Gamma_{2}$ the forces $F_{f, p, 2}^{i}$ and moments $M_{f, p, 2}^{i}$ can be computed as follows:
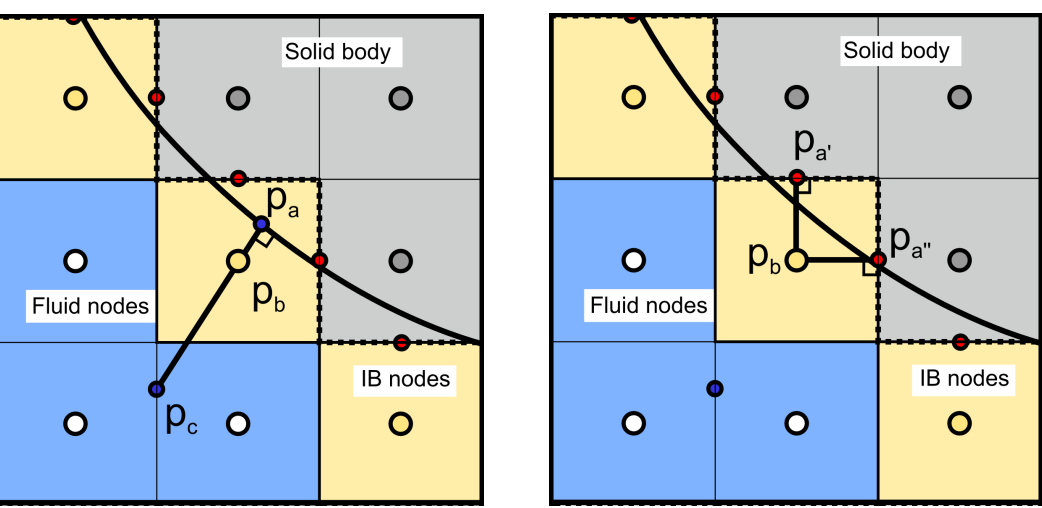

Figure 3: Schematic description of the successive pressure projections used to calculated the pressure on $\Gamma_{2}$ in the proposed PPBC method: step 1 (left) and step 2 (right).

The implementation of the PBBC approach is straightforward in methods such as the HCIB method or the CURVIB method in which a similar algorithm is used for reconstructing the velocity boundary condition at the IB nodes. Also, since the integration of the stresses is carried out in the background mesh, the parallel implementation of the algorithm is significantly simpler than in methods based on projecting the stresses to the unstructured Lagrangian mesh of the body. The simplicity and expedience of both algorithms, i.e. the PPBC and that from [21], when compared to methods based on projecting directly on the body surface, is in expense of an additional error caused by the approximation of the surface area. In the following we discuss the relative accuracy of the two methods. 


\subsubsection{Truncation error analysis}

Whether we apply the method of [21] or the proposed PPBC method, the accuracy of the calculated forces and moments on the body depends on the accuracy of the pressure field and the accuracy of the approach used to approximate the surface of the body. As far as the pressure is concerned, both methods employ the same fractional-step approach to calculate the pressure field. It is well known that fractional step algorithms yield first-order accurate pressure field [35] and as such both the method of [21] and the PPBC employ similar accuracy pressure fields. We note, however, that in problems with floating or fully submerged bodies at equilibrium, when the force on the body is dominated by the hydrostatic pressure, a first order approximation of the pressure can accurately represent the linear variation of the pressure field. Therefore, for such problems the accuracy of the two approaches will be determined by the relative accuracy of the approach each method employs to approximate the surface of the body.

For the method of Borazjani et al. [21], it can be readily shown that the error in approximating the surface area of the body is exactly first order. This is due to the fact that this method approximates the volume of the body $V_{\Omega}$ by a larger volume $V_{\Omega_{1}}$. Assuming that the layer of IB nodes surrounding the body has thickness of order $\Delta h$, an approximate expression for $V_{\Omega_{1}}$ can be written as follows:

$$
V_{\Omega_{1}}=V_{\Omega}+S_{\Gamma} \Delta h+H . O . T
$$

where $S_{\Gamma}$ is the surface area of the body and H.O.T indicates terms that are higher order in $\Delta h$. Since $\Delta h$ is of order $\Delta x$, equation (26) suggests that the method of [21] for calculating the force is first order accurate in space.

Calculating the accuracy of the body volume approximation in the PPBC method turns out to be somewhat more involved than for the method of [21]. This is because the computation of the volume in the PPBC as the sum of quadrilateral cells that cover the interior of the body is equivalent to the well known problem of calculating the area within a closed convex curve $C$ using lattice points [36, 37]. To demonstrate the underlying concepts and for the sake of simplicity we consider the $2 \mathrm{D}$ equivalent of the problem, namely the calculation of the area $\mathrm{S}$ of a closed curve as a summation of Cartesian grid cells forming a lattice. In such a case the surface $S$ can be written as follows:

$$
S=N \Delta h^{2}-E
$$

where $N$ is the number of lattice points located in the interior of the curve, $\Delta h$ is the spacing of the lattice points (equal to the grid spacing), and $E$ is the error of the approximation. Several authors have demonstrated the existence of bounds that limit the error $E$ (see for example [36]). These bounds, which depend on the curvature of the boundary, can be expressed in the following form:

$$
E \leq \frac{C}{\Delta h^{K-2}}\left[\log \left(\frac{1}{\Delta h}\right)\right]^{M}
$$

The study of Van der Corput [38] demonstrated that for piece-wise curves of class $C^{2}$ (a curve is of class $C^{r}$ when its radius of curvature is nonzero and $r-2$ times continuously differentiable with respect to the curve tangent direction) an upper limit of the error bound is (28) with values of $K$ and $M$ equals to $2 / 3$ and 0 , respectively. In such case the 
error in the computation of the surface is of order $O\left(\Delta h^{4 / 3}\right)$. For curves of class $C^{3}$ Huxley [36] obtained $K=131 / 208$ and $M=131 / 8320$. The classical example of application of this type of lattice point methods is the so-called Gauss's circle problem, which is schematically represented in figure 4 . The error bound of [36] for $C^{3}$ curves also applies for this problem. For an extensive review of lattice point methods applicable to other type of boundaries, such as boundaries with zero curvature points, or higher dimension boundaries, the reader is referred to the work of Ivic et al. [37].

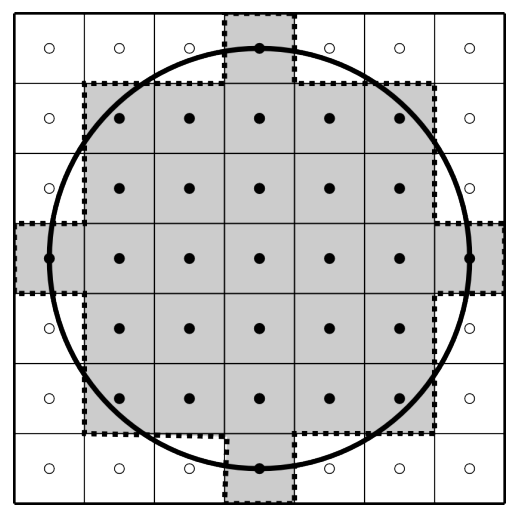

Figure 4: Schematic description of the Gauss's circle problem for the case of a unit radius circle and $\Delta h=1 / 3$.

Therefore, and based on the above analysis, we can estimate the order of accuracy of the volume calculation method used in the PPBC to be better than first order. Consequently we also anticipate that the PPBC will yield more accurate results in the calculation of the buoyancy force than the method of [21]. We will revisit this issue in section 4.2 where we will present a numerical accuracy test that confirms the theoretical arguments presented in this section.

\subsubsection{Implementation for arbitrarily complex bodies}

The PPBC method can, in principle, be applied to study one or more bodies of any general shape. However, in particular cases, such as in concave shaped bodies, or when two or more surfaces are located in the proximity of each other, numerical difficulties may arise requiring special attention. In fact, the same difficulties are also encountered in the reconstruction step of the CURVIB method (see section 3.4 below for a brief description of the CURVIB method). As sketched in figure 5, this problem can be observed when the wall normal vector passing through the IB node $b$ remains within the layer of IB nodes and does not extend into the area of fluid nodes from where flow variables can be extracted to reconstruct the pressure and/or velocity at the IB node $b$. Note that in both procedures, i.e. the first step of the proposed PPBC method and the velocity reconstruction in CURVIB, we employ the exact same wall normal vector as well as the same auxiliary point $c$. In such cases, neither the velocity nor the pressure can be obtained at point $c$, and thus, the method cannot proceed. Such difficulties, however, are encountered only when the background grid is too coarse to represent the shape of the body. As illustrated in figure $5 \mathrm{~b}$, with sufficient grid refinement this situation is alleviated. Therefore, preventing a situation such as that discussed above from occurring requires using a grid that is sufficiently fine to accurately represent regions of concave curvature of the body under consideration. 


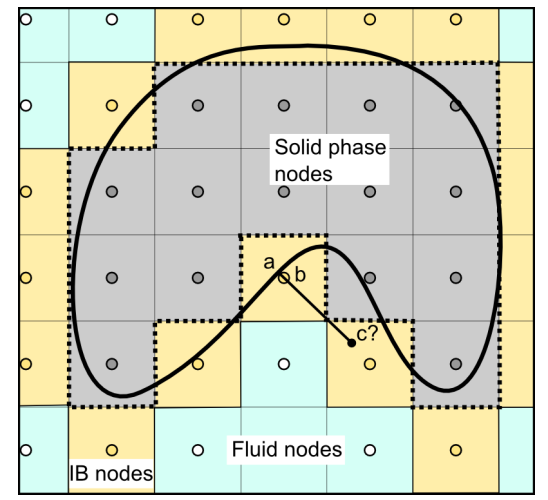

(a) Continuous concave surface, coarse grid

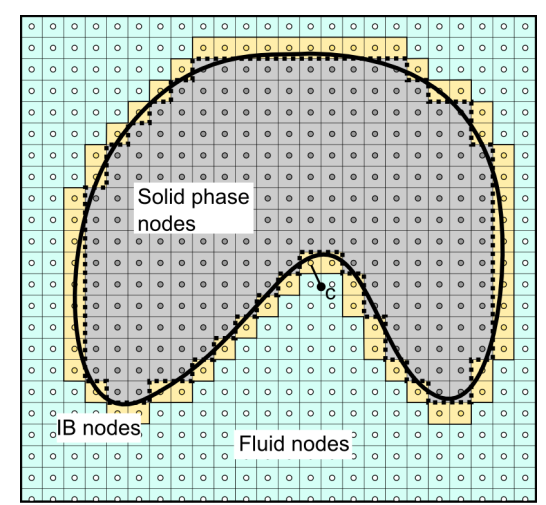

(b) Continuous concave surface, finer grid

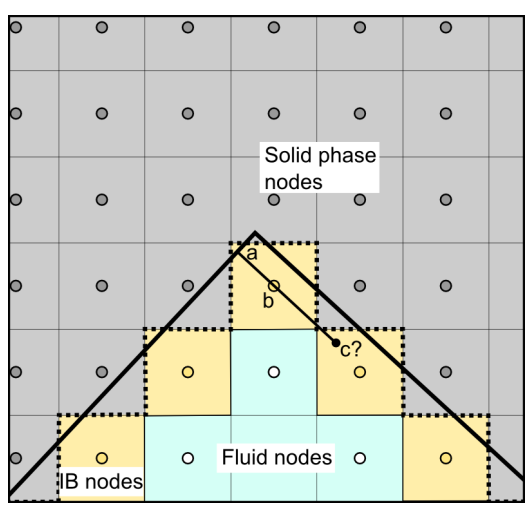

(c) Discontinuous surface

Figure 5: Schematic description of problems arising in the implementation of the PPBC and CURVIB methods to handel concave surfaces. a, b) A continuous concave surface for which difficulties can be alleviated by grid refinement; c) A discontinuous surface for which a special treatment is required.

Another difficulty in implementing the PPBC (as well as in performing the velocity reconstruction for the CURVIB method) arises when two or more adjacent surfaces intersect to form sharp angles, as illustrated in figure 5c. In such a case, the surface discontinuity cannot be eliminated even with grid refinements as there will always be at least one point (located near the intersection of the two surfaces) for which the velocity/pressure reconstruction will not be with the general approach. The only practical approach to handle such singular points is to implement a special treatment, based on interpolating the information from the closest fluid cells.

\subsection{The FSI-CURVIB method}

The method for tracking the motion of geometrically complex bodies is the sharp interface CURVIB method of Ge and Sotiropoulos [33], which has been thoroughly validated for simulating deformable bodies with large motions in various applications, including FSI problems [21, 39]. For the sake of completeness only a brief description of the method is presented herein. In the CURVIB approach, the body is represented by an unstructured triangular mesh which is embedded in the background curvilinear or Cartesian grid. An efficient searching algorithm is used to classify all nodes of the computational domain depending in their location with respect to the position of the body as already discussed in section 3.3. The linking between the background and structure grids is done through a sharp interface approach by reconstructing the boundary conditions for the velocity field and the distance function $\phi$ at the IB nodes (see Ge and Sotiropoulos [33] and Kang and Sotiropoulos [32] for details). The velocity is reconstructed in the wall normal direction with either linear or quadratic interpolation in the case of low Reynolds number flows when the IB nodes are located in the viscous sub-layer, or using the wall models described by $[40,41,42]$ in high Reynolds number flows when the grid resolution is not sufficient to accurately resolve the viscous sub-layer. The distance function $\phi$ is 
reconstructed by setting its gradient to be zero at the cell faces that are located between the fluid and IB nodes. This is equivalent to applying a zero Neumann boundary condition along the grid line corresponding to the aforementioned cell faces. A further description of how to reconstruct the distance function in the IB nodes is given in [32].

The equations of motion (Eqs. 9 and 10) are coupled with the fluid domain equations through a partitioned FSI approach. The time integration can be done explicitly with loose coupling (LC-FSI), or implicitly with strong coupling (SC-FSI). The Aitken acceleration technique of [43] is able to significantly reduce the number of sub-iterations when the SC-FSI algorithm is used. A detailed description of both time-integration algorithms is given in [21].

\subsection{Solution Procedure}

The procedure for solving the overall problem using the SC-FSI approach is summarized as follows.

1. Starting with an initial condition or previous time step values for the fluid domain $\left(\phi^{n}, \mathbf{U}^{n}, p^{n}\right)$ and the structural domain $\left(\mathbf{X}^{n}, \Theta^{n}\right)$ variables, advance the solution as follows.

2. For $k=1$, set $\widetilde{\mathbf{U}}^{k}=\mathbf{U}^{n}, \widetilde{p}^{k}=p^{n}, \widetilde{\mathbf{X}}^{k}=\mathbf{X}^{n}$, and $\widetilde{\mathbf{\Theta}}^{k}=\boldsymbol{\Theta}^{n}$ and start a sub iteration loop.

3. Calculate the forces and moments using Eqs. (17) and (18) $\widetilde{\mathbf{F}}_{\text {Fluid }}^{k+1}=\widetilde{\mathbf{F}}_{\text {Fluid }}^{k+1}\left(\phi^{n}, \widetilde{\mathbf{U}}^{k}, \widetilde{p}^{k}, \widetilde{\mathbf{X}}^{k}, \widetilde{\boldsymbol{\Theta}}^{k}\right)$ and $\widetilde{\mathbf{M}}_{\text {Fluid }}^{k+1}=$ $\widetilde{\mathbf{M}}_{\text {Fluid }}^{k+1}\left(\phi^{n}, \widetilde{\mathbf{U}}^{k}, \widetilde{p}^{k}, \widetilde{\mathbf{X}}^{k}, \widetilde{\boldsymbol{\Theta}}^{k}\right)$.

4. Solve the equations of motion (9-10) to obtain the new position of the structure $\widetilde{\mathbf{X}}^{k+1}=\widetilde{\mathbf{X}}^{k+1}\left(\widetilde{\mathbf{X}}^{k}, \widetilde{\boldsymbol{\Theta}}^{k}, \widetilde{\mathbf{F}}_{\text {Fluid }}^{k+1}\right.$ ) and $\widetilde{\boldsymbol{\Theta}}^{k+1}=\widetilde{\boldsymbol{\Theta}}^{k+1}\left(\widetilde{\mathbf{X}}^{k}, \widetilde{\boldsymbol{\Theta}}^{k}, \widetilde{\mathbf{M}}_{\text {Fluid }}^{k+1}\right)$.

5. If $k=1$, solve the Level set and reinitialization equations (7) and (8) to obtain $\phi^{n+1}=\phi^{n+1}\left(\phi^{n}, \mathbf{U}^{n}, \mathbf{X}^{k+1}, \Theta^{k+1}\right)$.

6. Solve the momentum and Poisson equations (2) and (13) to obtain new values for the flow variables $\widetilde{\mathbf{U}}^{k+1}=$ $\widetilde{\mathbf{U}}^{k+1}\left(\phi^{n+1}, \widetilde{\mathbf{U}}^{k}, \widetilde{\mathbf{X}}^{k+1}, \widetilde{\boldsymbol{\Theta}}^{k+1}\right)$ and $\widetilde{p}^{k+1}=\widetilde{p}^{k+1}\left(\phi^{n+1}, \widetilde{\mathbf{U}}^{k}, \widetilde{\mathbf{X}}^{k+1}, \widetilde{\boldsymbol{\Theta}}^{k+1}\right)$.

7. Check the convergence of the structural position: $\left\|\widetilde{\mathbf{X}}^{k+1}-\widetilde{\mathbf{X}}^{k}\right\|<\epsilon_{0}$ and $\left\|\widetilde{\boldsymbol{\Theta}}^{k+1}-\widetilde{\boldsymbol{\Theta}}^{k}\right\|<\epsilon_{0}$.

8. If convergence has been achieved, advance to step 1 , otherwise increment $\mathrm{k}$ by one and return to step 3 .

The LC-FSI algorithm is derived by the above algorithm by setting $\mathrm{k}=\mathrm{n}$ and performing only one FSI iteration per time stepping, essentially stopping the algorithm at step 6 above and advancing to the next time step. Note that in the above SC-FSI algorithm the level set equation is solved only once per time step. Numerical tests showed that incorporating this equation in the SC sub-iteration loop increases the computational time without any quantifiable benefit in the accuracy of the computed solution.

\section{Results}

In this section, we apply the coupled CURVIB, level set, FSI algorithm to simulate a number of test cases of gradually increasing complexity. We seek to demonstrate the accuracy of the method by comparing its results with previous numerical studies and experimental measurements, probe via numerical experiments various aspects of the proposed computational algorithm, and demonstrate the ability of the method to simulate complex FSI problems involving geometrically complex structures interacting with the free surface. 
Table 1: Description of the four grids employed in the water entry/exit case of a horizontal circular cylinder.

\begin{tabular}{ccc}
\hline Grid & Grid size (horizontal, vertical) & Near cylinder spacing \\
\hline \hline 1 & $170 \times 125$ & $0.2 \mathrm{R}$ \\
2 & $220 \times 160$ & $0.1 \mathrm{R}$ \\
3 & $360 \times 255$ & $0.05 \mathrm{R}$ \\
4 & $640 \times 450$ & $0.0025 \mathrm{R}$ \\
\hline
\end{tabular}

\subsection{Prescribed motion test case: Water entry/exit of a horizontal circular cylinder}

The first test case was selected to validate the CURVIB level set method and involves a body moving across the air/water interface with prescribed motion. Therefore, the FSI algorithm is not employed in this test. We consider the two $2 \mathrm{D}$ cases of an infinitely long cylinder of radius $\mathrm{R}=1$ moving with constant speed in an infinitely wide domain downward (upward) crossing the free surface from the air (water) phase. We shall refer to the first case as the entry (from air to the water) problem and to the second case as the exit (from the water to the air) problem. In the entry case, the configuration we study herein is identical to that simulated by [44, 24], where an identical cylinder, initially positioned above the free surface at a distance $h=1.25$, moves downwards with constant velocity of $u=-1$. The water density is $\rho_{\text {water }}=1$ and the dynamic viscosity $\mu_{\text {water }}=1 \cdot 10^{-3}$ while the corresponding values for air are $\rho_{\text {air }}=1 \cdot 10^{-3}$ and $\mu_{\text {air }}=1.8 \cdot 10^{-5}$. The gravity is set to $g=-1$ and the time is normalized as $T=u t / h$.

The 2D computational domain is $40 R \times 24 R$ in the horizontal and vertical directions, respectively, as in [24]. Four non-uniform, successively finer meshes were employed to systematically investigate the grid sensitivity of the computed solutions. Each grid consists of an inner region, centered on the cylinder, within which the mesh is uniform and an outer region where the grid is gradually stretched. For all grids the inner region is the rectangular domain defined by $[-5,5]$ and $[-4,2.6]$ in the horizontal and vertical directions, respectively. Within this inner domain uniform grid spacing is employed along both directions, which is equal to $0.2 R$ for grid $1,0.1 R$ for grid $2,0.05 R$ for grid 3 , and $0.025 R$ for grid 4 . Outside of this inner domain the mesh is stretched gradually away from the cylinder using the hyperbolic stretching function with a stretching ratio kept below 1.05. The number of nodes for each mesh is: $170 \times 125,220 \times 160,360 \times 255$, and $640 \times 450$ for grids 1 to 4 , respectively. A summary of the grids we employ is given in table 1.

The free surface and flow patterns calculated on grid 3 with a time step of 0.01 are presented at different instances in time in figure 6, which shows the calculated free surface position, cylinder position and induced vorticity field. It is evident from this figure that as the cylinder impinges onto the free surface two inclined jets of water form on each side of the cylinder where the free surface steepens and ultimately breaks (see figure $6 \mathrm{~d}$ ). As the cylinder continues its motion through the surface it creates a system of waves that propagate outward away from the body. Ultimately the cylinder enters fully the water phase and its downward motion is seen to create a steep, vertical cusp in the free surface marking the trailing jet of the water in the cylinder wake (see figures $6 \mathrm{~h}$ and i). At all instances in time vorticity is 


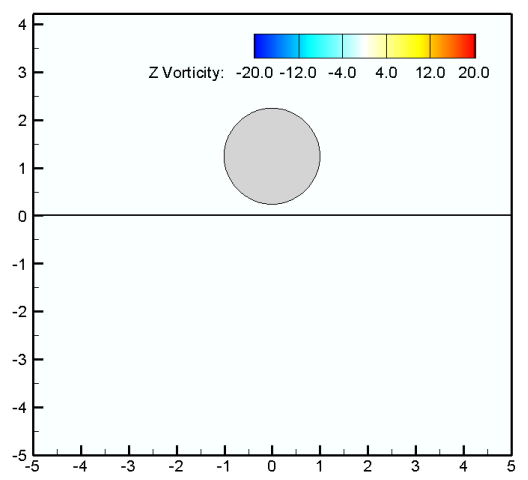

(a) $\mathrm{T}=0.0$

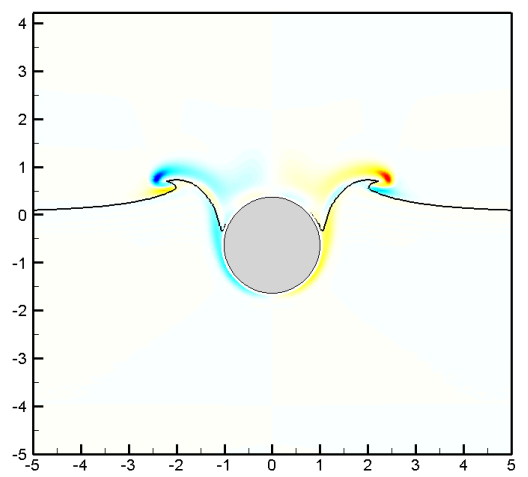

(d) $\mathrm{T}=1.5$

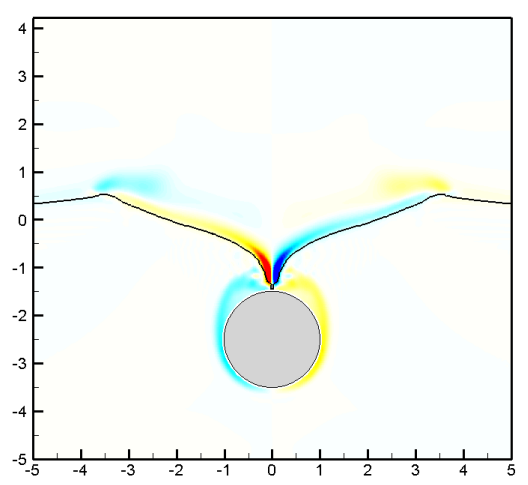

(g) $\mathrm{T}=3.0$

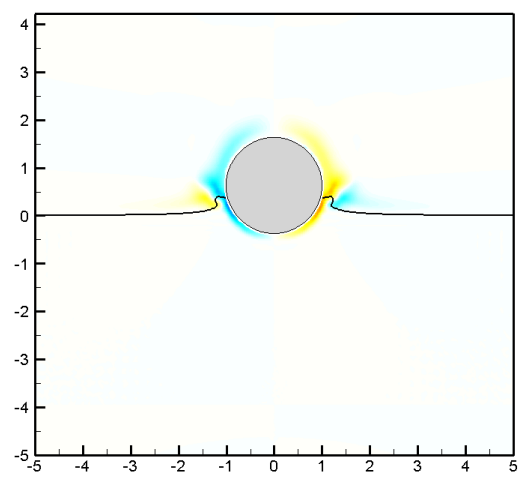

(b) $\mathrm{T}=0.5$

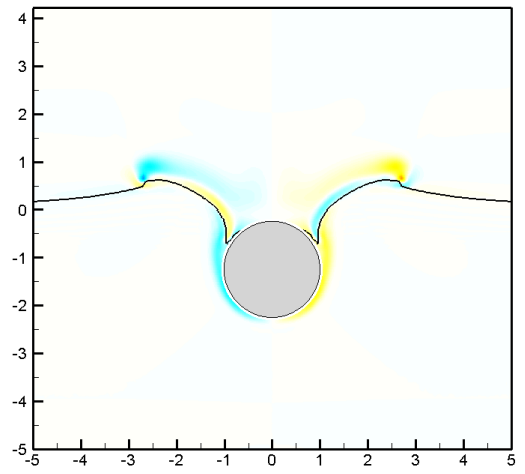

(e) $\mathrm{T}=2.0$

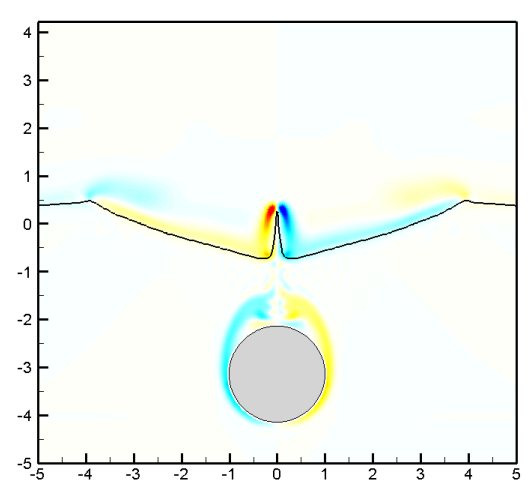

(h) $\mathrm{T}=3.5 \mathrm{~s}$

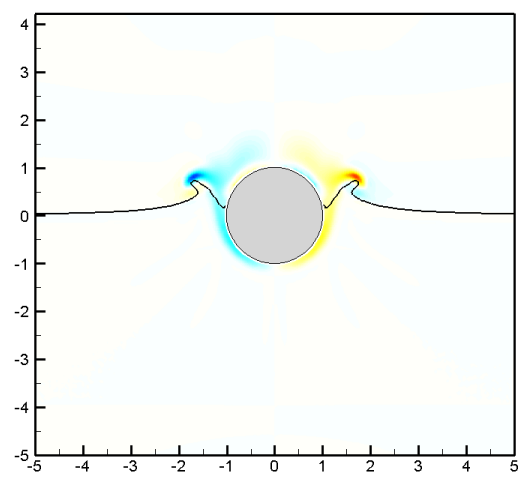

(c) $\mathrm{T}=1.0$

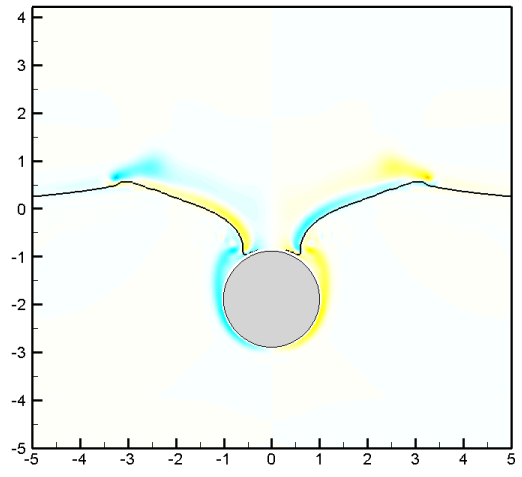

(f) $\mathrm{T}=2.5$

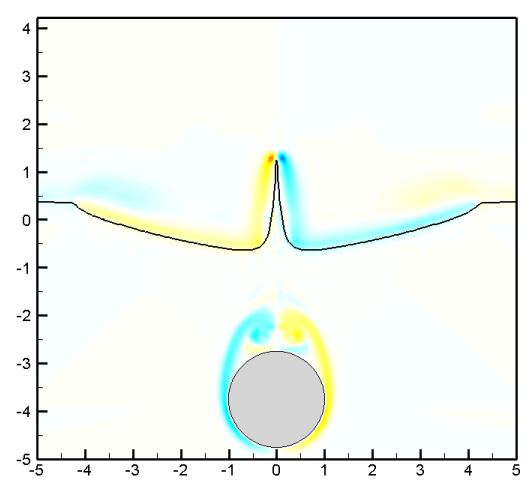

(i) $\mathrm{T}=4.0 \mathrm{~s}$

Figure 6: Water entry of a horizontal circular cylinder moving with prescribed velocity. Simulated free surface position at different times along with vorticity contours. The results have been obtained on grid 3 with a time step of 0.01 .

generated in the vicinity of the cylinder but also across the air-water interface in regions where curvature develops in the free surface.

The free surface patterns captured by our simulation on grid 3 are compared with those reported by [24] who employed a grid of similar resolution (figure 7). More specifically [24] employed a non-uniform two-region grid of 


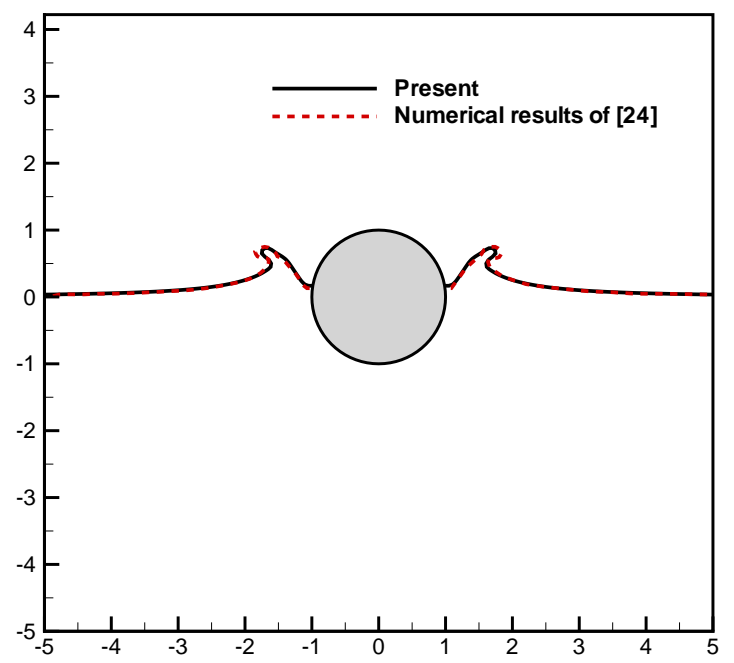

(a) $\mathrm{T}=1$

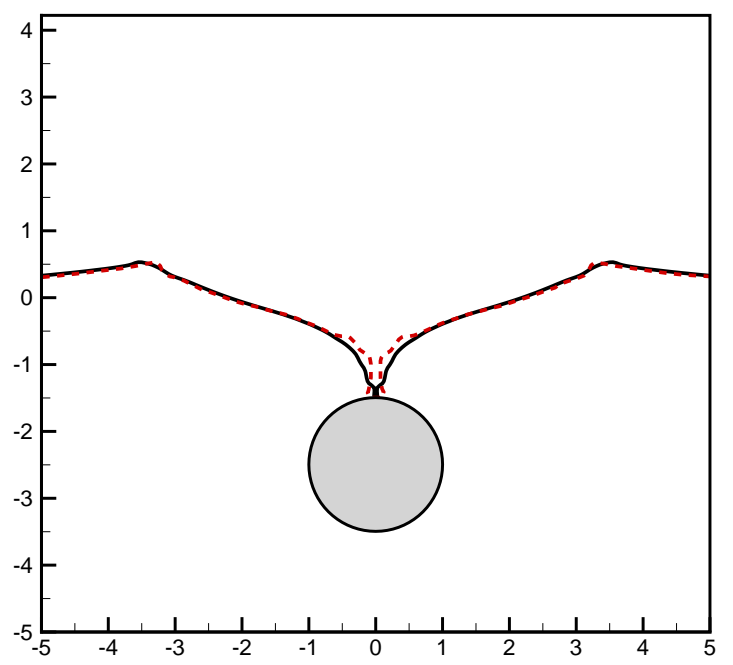

(c) $\mathrm{T}=3$

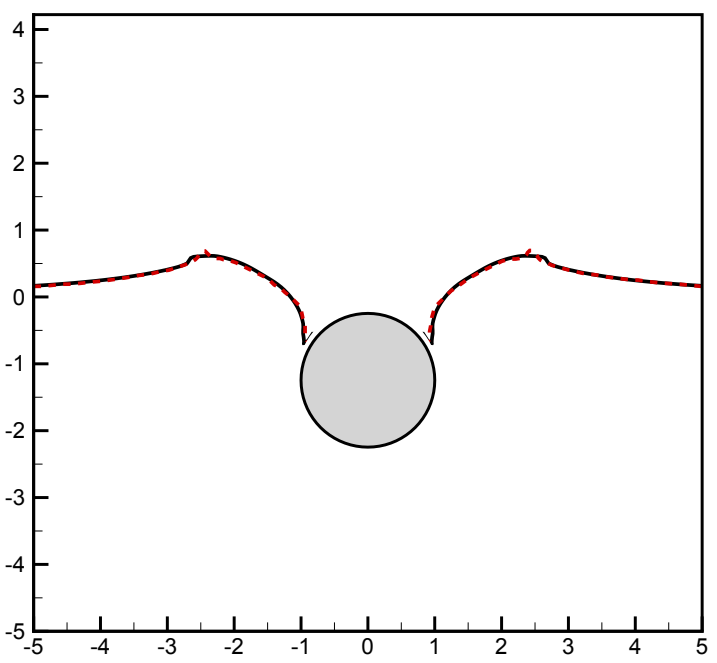

(b) $\mathrm{T}=2$

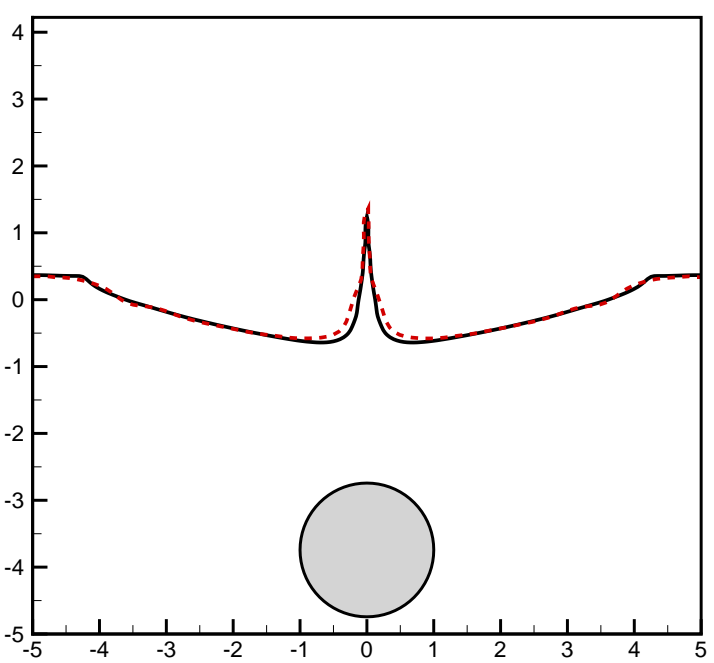

(d) $\mathrm{T}=4$

Figure 7: Water entry of a horizontal circular cylinder moving with prescribed velocity. Free surface position at different non-dimensional times $T$ calculated by the present method and the method of Yang et al. [24]. The results have been obtained on grid 3 with a time step of 0.01 .

size $300 \times 240$ with uniform inner rectangular domain centered on the cylinder with the same grid spacing of $0.05 \mathrm{R}$.

It is evident from this figure that our results are in good agreement with those obtained by [24]. However, minor discrepancies are observed at times $T=3$ and $T=4$ which can be explained by the fact that [24] employed a sharp interface level set method and a contact angle boundary condition while the present method uses a diffused interface method and no contact angle boundary condition. Treating the air/water interface with a sharp method results in overall higher accuracy as the actual pressure jump condition is taken directly in consideration. On the other hand, diffused methods are in general simpler to implement and the introduction of a diffusion thickness allows the method 


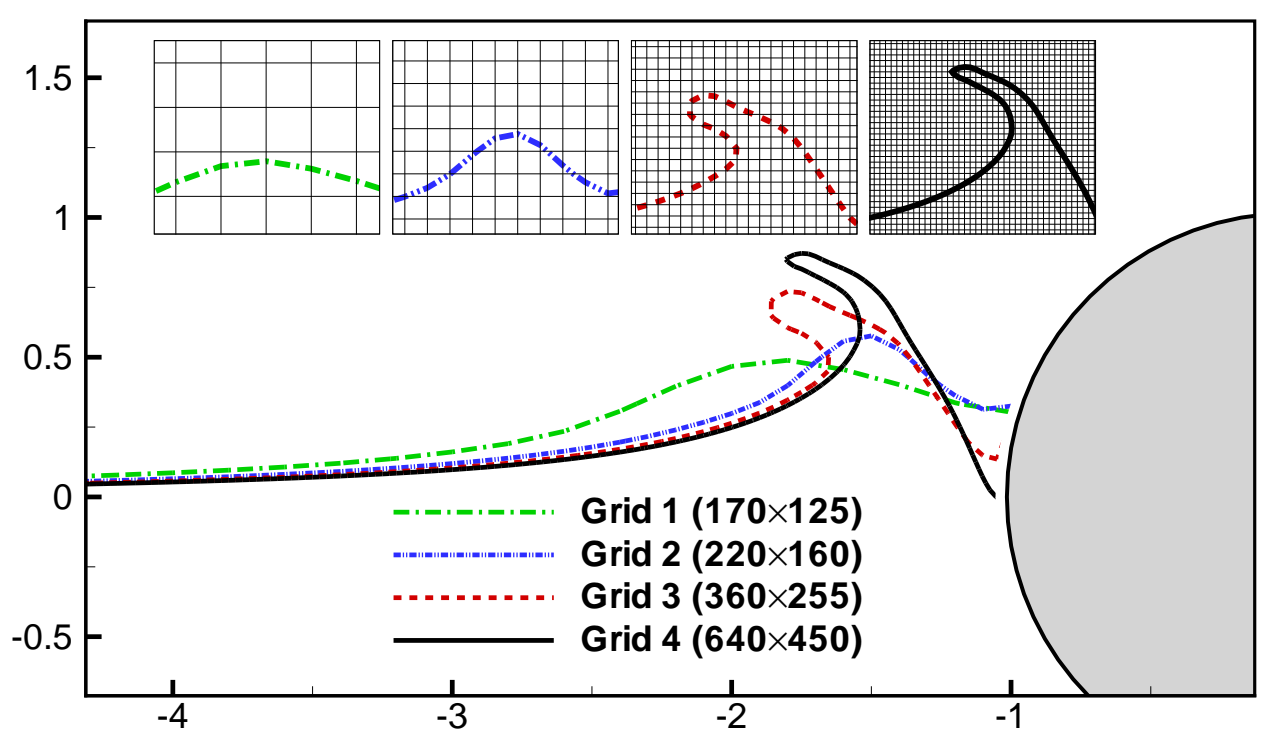

Figure 8: Water entry of a horizontal circular cylinder moving with prescribed velocity. Influence of the grid refinement on the free surface position at time $T=1.0$ computed with a time step of 0.005 .
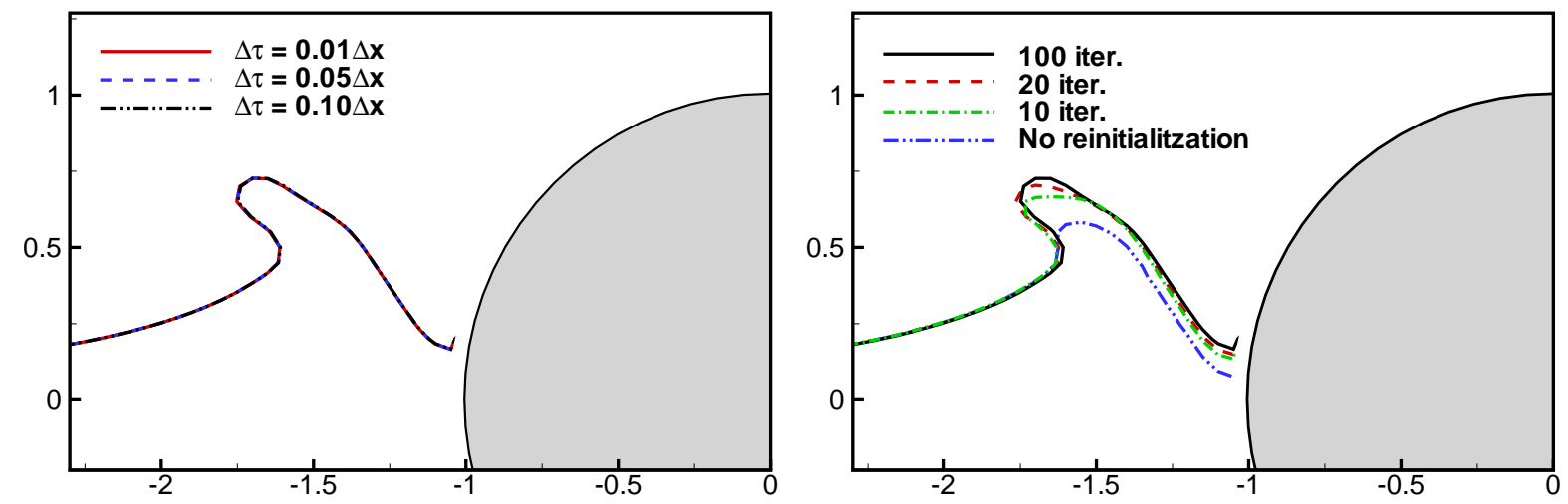

Figure 9: Water entry of a horizontal circular cylinder moving with prescribed velocity. Influence of the reinitialization time step $\Delta \tau$ (left) and the pseudo-time interval $\tau$ (right) on the accuracy of the calculated free surface position at time $T=1.0$ computed on grid 3 . The left figure shows the free surface when the full interface thickness is reinitialitzed using different time step sizes $\Delta \tau$. The figure on the right shows different levels of reinitialization for a constant step size of $\Delta \tau=0.01 \Delta x$. For both cases the interface thickness is $\epsilon=\Delta x$, therefore when $\Delta \tau=0.01 \Delta x$ the interface is fully reinitialized with 100 iterations.

to prevent the formation of disturbances which may originate with the presence of an abrupt jump as discussed in [45].

To demonstrate the sensitivity of the computed results to grid refinement we show in figure 8 the predicted free surface shape on all four grids at an instant in time when the water jet off the side of the cylinder steepens just before the wave breaks. The same time step size of 0.005 and an interface thickness $\epsilon$ of 0.04 was employed for all grids. A number of important conclusions are obtained from this figure. First, it is evident that only the two finest meshes, 


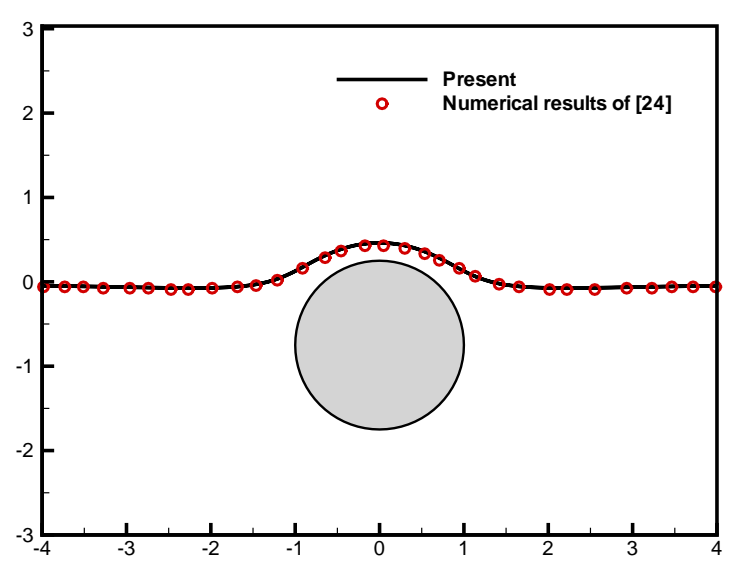

(a) $\mathrm{T}=0.4$

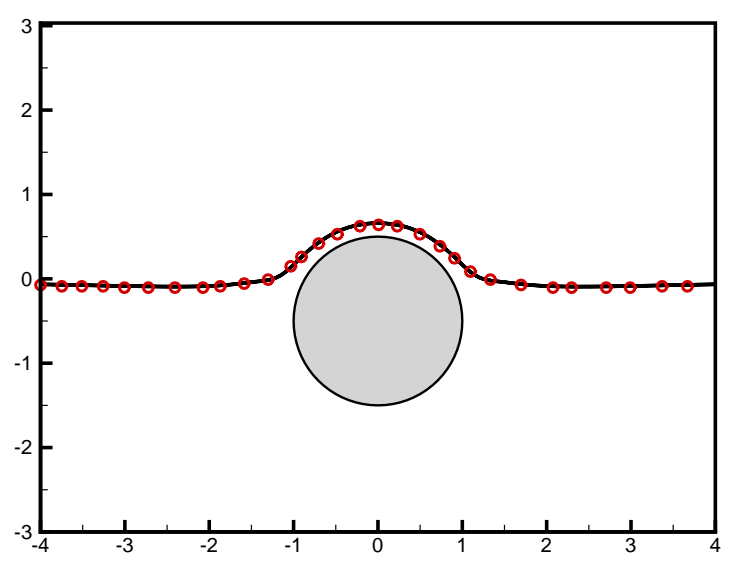

(b) $\mathrm{T}=0.6$

Figure 10: Water exit of the horizontal circular cylinder moving with prescribed velocity. Free surface position at different non-dimensional times $T$ calculated by the present method and the method of Yang et al. [24]. The results have been obtained on grid 3 with a time step of 0.005 .

grids 3 and 4, are able to capture the steepening and folding of the free surface that ultimately leads to wave breaking. The two coarser meshes fail completely to resolve this feature of the flow. Second, within the region of steep surface curvature the solution is clearly not grid insensitive since there is considerable difference between the calculated free surface shapes on grids 3 and 4. More specifically, as the grid is refined the surface becomes steeper, reaches higher elevation, and a much sharper cusp develops at the wave front. It is expected, however, that as the surface steepens and begins to fold phenomena at continuously finer scales will emerge, ultimately leading to the formation of a very thin film, and a much finer mesh will be required to resolve them. This finding notwithstanding, however, it is also evident from figure 8 that away from the near-cylinder region where the interface steepens and folds grids 3 and 4 yield similar results. A common feature observed in all four grid cases is the formation of a wave that grows and steepens up to a certain degree depending on the grid resolution, although in the case of grids 1 and 2 the resolution is not sufficiently fine to capture the wave folding and breaking. Looking at the insets in the same figure 8 showing the wave pattern along with the mesh, it is clear that the wave stops steepening when the crest is resolved by approximately four grid cells. If we consider as the characteristic length of the phenomena half of the wave length (approximately equal to the size of the inset window size in figure 8), we note from figure 8 that this length is discretized with $6,12,24$ and 48 grid nodes in grids 1 to 4 respectively. It is evident that grids 1 and 2 fail to capture the wave steepening, which only arises for the first time on grid 3. We can thus conclude that resolving with the present method wave steeping and folding phenomena requires at least 24 grid nodes per one half of the wavelength.

The influence of the level set reinitialization pseudo-time step size $\Delta \tau$ and the total re-initialization time $\tau$ in Eqn. (8) is illustrated in figure 9, which shows the calculated free surface patterns on grid 3. When the thickness of the interface layer is fully reinitialized, i.e. $\tau=\epsilon$ (see discussion in section 3.2 above), and different re-initialization time step sizes are used to solve Eqn. (8), $\Delta \tau=0.01 \Delta x, 0.05 \Delta x$ and $0.10 \Delta x$, it is evident from figure 9a that the resulting 
free surface shapes are identical. Note here that since $\epsilon$ is equivalent to one grid cell spacing, to fully reinitialize the above cases it was necessary to perform 100,20, and 10 reinitialization iterations, respectively. Clearly, therefore, the computed solutions are not sensitive to the size of the re-initialization time step as long as the condition $\tau=\epsilon$ is satisfied. As shown in the figure $9 \mathrm{~b}$, however, the computed free surface pattern is very sensitive to the total reinitialization time. This figure compares the free surface shape at the same instant in time using the same reinitialization time step $\Delta \tau=0.01$ but progressively longer re-initialization times: from no re-initialization $\left(n_{\tau}=0\right)$ to full re-initialization $\left(n_{\tau}=100\right)$. It is evident from this figure that the result without re-initialization is not accurate but as the re-initialization time increases the computed solution converges monotonically toward the fully re-initialized solution. Moreover, it is also evident that using between 10 to 20 re-initialization iterations, which is equivalent to a reinitialization covering $10-20 \%$ of the interface thickness, is sufficient to obtain a solution that is very close to the fully converged solution. The non-reinitialized case shows large discrepancies when compared to the fully one. This is an important result to consider when simulating complex free surface interface phenomena, which require the use of large thickness $\epsilon$ of the strip in conjunction with small step size $\Delta \tau$. For such cases, full re-initialization would be very expensive but based on the results reported in figure $9 \mathrm{~b}$ re-initializing the solution within a region covering $10-20 \%$ of the interface thickness should provide a good compromise between accuracy and computational efficiency. We also have to consider that using a large interface thickness $\epsilon$ introduces and additional source of error. To understand the sensitivity of the interface thickness to the flow solution the reader is referred to the work of Iafrati and Campana [28]. It was shown that the use of a large interface thickness does not prevent the model from obtaining accurate dynamics of the free surface and the vorticity field of the flow.

Finally, the water exit case for the cylinder problem we simulate herein corresponds to the same configuration as in [46] or [24]. Compared to the previously discussed case, the difference is that the cylinder is initially positioned to be fully submerged underwater at a distance $h=-1.25$ below the free surface and its prescribed velocity is upward and equal to $u=0.39$. This case has been simulated on grid 3 with a time step size of 0.005 and a free surface interface thickness of 0.03. Figure 10 shows comparisons between our simulations and the results of [24] for the free surface position at times $T=0.4$ and $T=0.6$. It is evident from this figure that agreement between the two simulations is excellent.

\subsection{Force calculation test case: Static buoyant cylinder case}

We employ herein two simple test cases to validate and demonstrate the accuracy of the PPBC method we proposed in section 3.3 for calculating the force acting on a floating structure. For case 1 a circular cylinder is partially submerged by half of its diameter while for case 2 the cylinder is completely submerged under water. The exact solution of both problems is known through the Archimedes' principle, namely the resultant buoyant force is equal to the weight of the displaced fluid. We employ the hydrostatic pressure field to calculate the buoyancy force using the standard method of Borazjani et al. [21] and the proposed PPBC method. Although this test case only evaluates the accuracy of the method we use to calculate the force due to pressure, it is highly relevant to our work since the method 

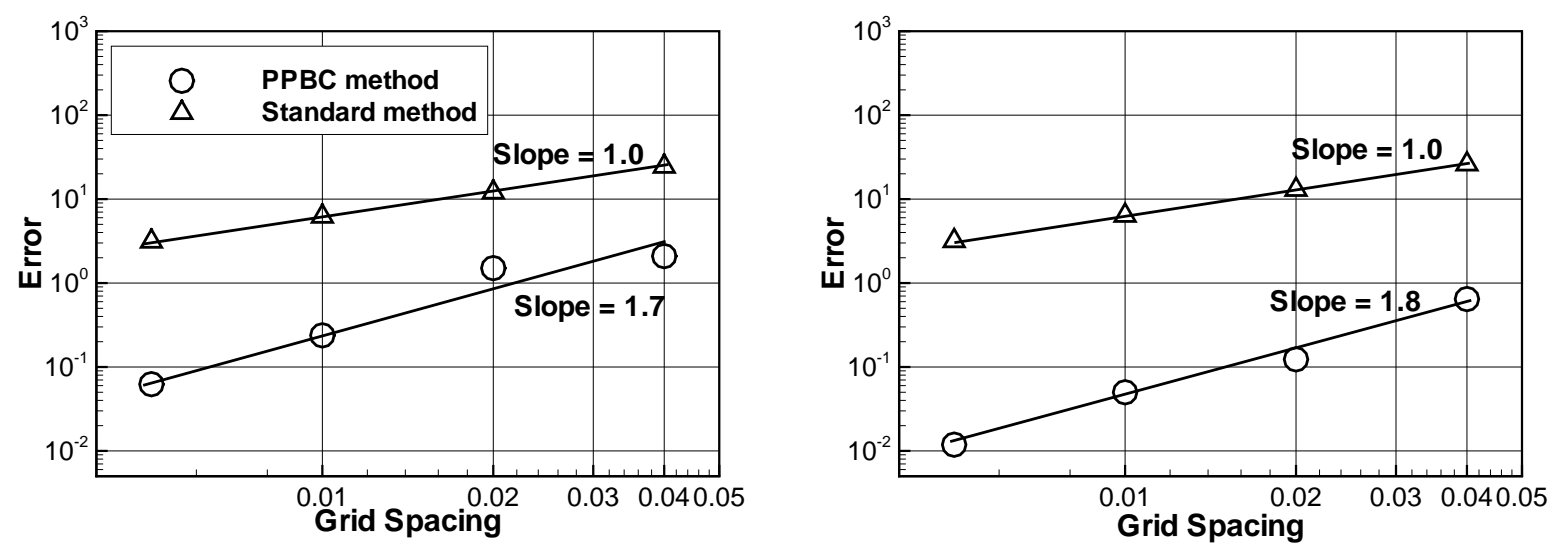

Figure 11: Static buoyant cylinder. Grid convergence of the error of the computed buoyant force due to pressure, for a partly (left) and fully (right) submerged static cylinder.

for calculating the pressure force is responsible for the numerical difficulties encountered when attempting to extend the FSI-CURVIB method of [21] to two-phase flow simulations. In addition, the pressure force is the main contributor to the total force acting on floating structures in offshore applications and as such the method for calculating it is of critical importance for developing an accurate FSI algorithm.

With reference to figure 11 , the buoyancy force calculated with the standard method (see [21]), $F=F_{S}$, is equal to the net force due to pressure computed by integrating along the surface $\Gamma_{1}$. When using the PPBC method, on the other hand, $\mathrm{F}$ is the resultant projected pressure force as described in section 3.3.2 in the surface $\Gamma_{2}$. For both cases we consider herein, the exact force is known and, therefore, the error between the two methods for calculating the force and the exact value can be defined as follows: $E=\left|F-F_{\text {exact }}\right| / F_{\text {exact }}$. In figure 11 we show in a log-log plot the convergence of this error calculated in a series of successively refined uniform meshes with spacing: $0.04 D, 0.02 D$, $0.01 \mathrm{D}$, and $0.005 \mathrm{D}$. It is evident from this figure that for both cases and all grids the standard method for calculating the force grossly over predicts the buoyancy force on the cylinder. Furthermore, while this method converges with grid refinement, the rate is very slow and the method is at best first-order accurate as expected from the analysis we presented in section 3.3.3. The proposed PPBC method, on the other hand, yields on all grids errors lower by nearly one order of magnitude than the standard method and its convergence rate is clearly better than first order for both cases -in fact the results in figure 11 suggest a nearly second-order convergence for the PPBC method. Therefore, the results in figure 11 confirm the theoretical arguments we presented in section 3.3.3 regarding the relative accuracy of the PPBC and the method of [21] for calculating the buoyancy force. The superior performance of the PPBC method will be further demonstrated in subsequent test cases where it will be used to solve complex FSI problems involving bodies interacting with a free surface. 


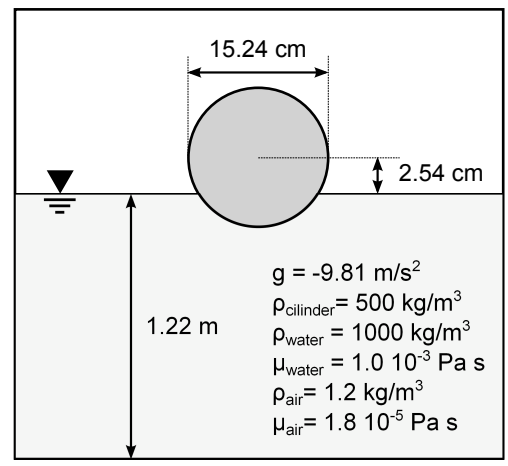

Figure 12: Free heave decay test of a circular cylinder. Schematic description of the cylinder configuration studied experimentally by Ito [47].

Table 2: Description of the four grids employed in the free heave decay test of a circular cylinder and the corresponding interface thickness $\epsilon$ employed.

\begin{tabular}{cccc}
\hline Grid & Grid size & Near cylinder spacing & Interface thickness $\epsilon$ \\
\hline \hline 1 & $320 \times 220 \times 8$ & $0.040 \mathrm{D}$ & $0.131 \mathrm{D}$ \\
2 & $440 \times 260 \times 8$ & $0.020 \mathrm{D}$ & $0.065 \mathrm{D}$ \\
3 & $680 \times 420 \times 8$ & $0.010 \mathrm{D}$ & $0.039 \mathrm{D}$ \\
4 & $1130 \times 720 \times 8$ & $0.005 \mathrm{D}$ & $0.039 \mathrm{D}$ \\
\hline
\end{tabular}

\subsection{FSI case 1: Free heave decay test of a circular cylinder}

With this and the subsequent presented test cases we seek to demonstrate the predictive capabilities of the coupled FSI, CURVIB, level set formulation for bodies of increasing complexity interacting with a free surface. We consider herein the same case as that studied experimentally by Ito [47], which is suited for validating the FSI algorithm in a translational single degree of freedom. A horizontal circular cylinder of diameter $D=0.1524 \mathrm{~m}$ and density $\rho=500 \mathrm{~kg} / \mathrm{m}^{3}$ is partially submerged with its center positioned $0.0254 \mathrm{~m}$ above the free surface of a rectangular channel (see figure 12 for a schematic representation). The computational domain is a $27.4 \mathrm{~m}$ long channel, $2.59 \mathrm{~m}$ wide, with a depth of $1.22 \mathrm{~m}$ and the water in it is initially stagnant. The cylinder movement is restricted to the vertical degree of freedom, and it is allowed to oscillate freely. The Reynolds number as defined in equation (3) based on the cylinder diameter and its maximum velocity is equal to $R e=30,000$. We assume that the cylinder spans the entire width of the channel and that the side walls are slip walls. We carry out 3D LES but using a coarse grid in the spanwise direction, that is, essentially assuming that the flow is 2D. Tests with finer grid in the spanwise direction did not yield any appreciable differences in the simulated response of the cylinder, which is the only quantity recorded experimentally. More specifically, we employ four non-uniform meshes: grid 1 with $320 \times 160 \times 8$; grid 2 with $440 \times 260 \times 8$; grid $3680 \times 420 \times 8$; and grid 4 with $1130 \times 720 \times 8$ nodes in the horizontal, vertical, and spanwise directions, respectively. The grids are uniform in a rectangular region centered around and containing the body defined by $[-0.3,0.3]$ in the horizontal direction and $[-0.2,0.2]$ in the vertical direction, and have a grid spacing equal to $0.04 D$, 


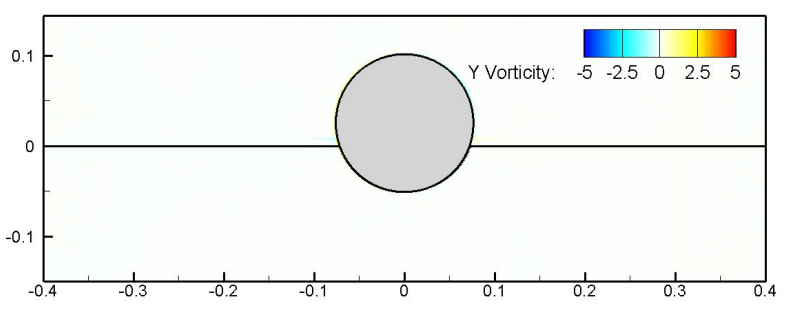

(a) $\mathrm{t}=0.0 \mathrm{~s}$

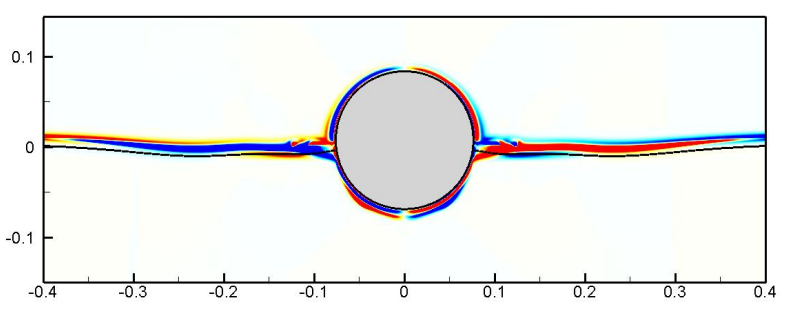

(c) $\mathrm{t}=0.6 \mathrm{~s}$

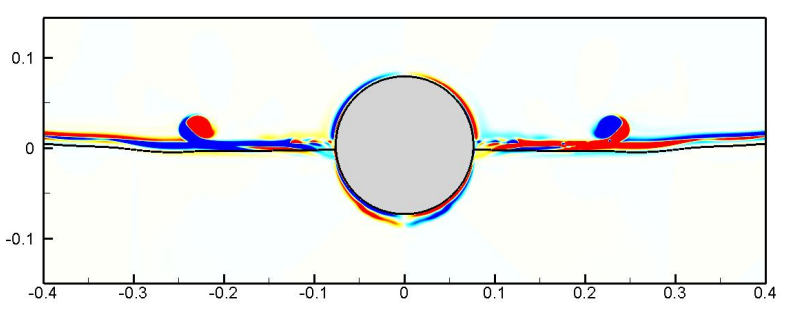

(e) $t=1.2 \mathrm{~s}$

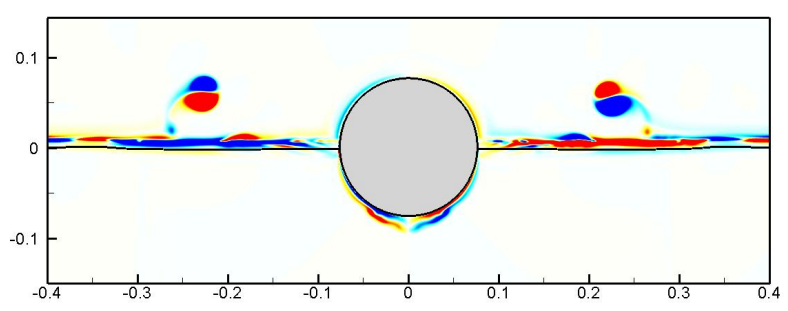

(g) $\mathrm{t}=1.8 \mathrm{~s}$

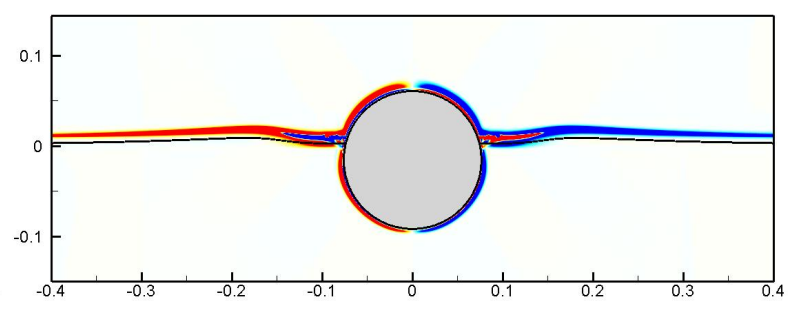

(b) $t=0.3 \mathrm{~s}$

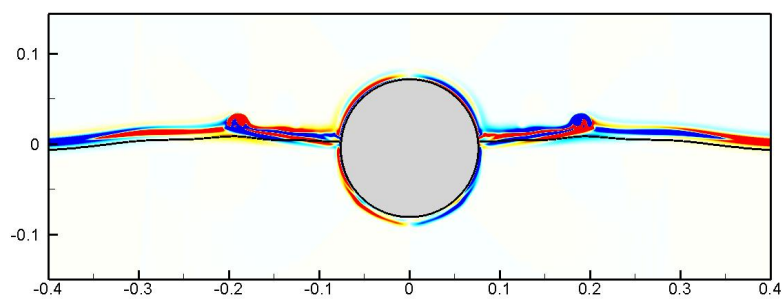

(d) $\mathrm{t}=0.9 \mathrm{~s}$

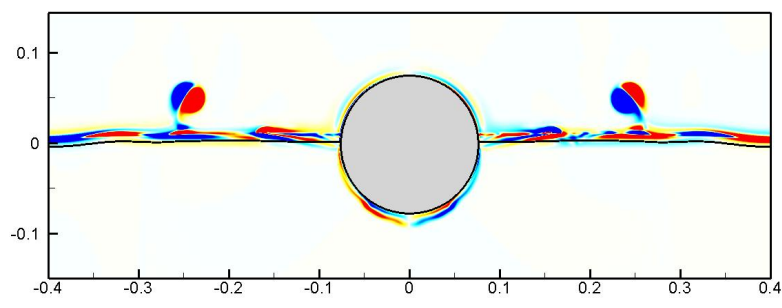

(f) $\mathrm{t}=1.5 \mathrm{~s}$

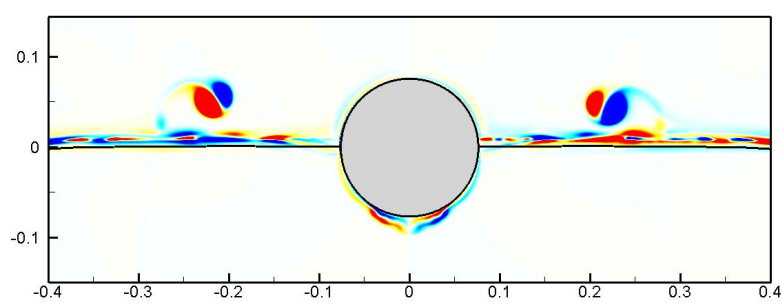

(h) $\mathrm{t}=2.1 \mathrm{~s}$

Figure 13: FSI simulation of heave decay of a circular cylinder. Several snapshots of the calculated position of the cylinder, the free surface, and corresponding out-of-plane vorticity contours are shown in these figures. The solution has been obtained on grid 4 , which has near-cylinder spacing equal to $\Delta x=\Delta y=0.005 D$, and a time step size of $0.0005 s$ has been used.

$0.02 D, 0.01 D$, and $0.005 D$ for grids 1 to 4 , respectively. In the domain outside this uniform grid region, the grids are stretched using a hyperbolic function and the ratio never exceeds 1.05. The grids description as well as the interface thickness $\epsilon$ used in each case are summarized in table 2. All simulations for this case were carried out using the LC-FSI algorithm and a time step size of $0.0005 s$.

Several snapshots on grid 4 of the calculated position of the cylinder and the free surface along with vorticity contours are presented in figure 13. It is seen in this figure that as the cylinder impinges on and begins to deform the 


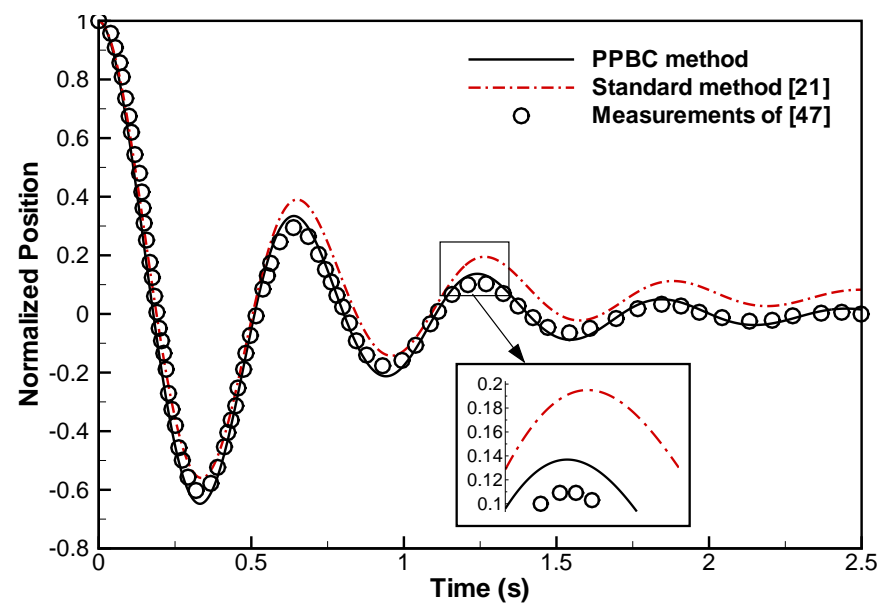

Figure 14: FSI simulation of heave decay of a circular cylinder. Normalized position of the cylinder computed on grid 3 with the PPBC method, the standard method, and the experimental data of Ito [47]. The time step size employed is $0.0005 \mathrm{~s}$ and the interface thickness $\epsilon$ is $0.006 \mathrm{~m}$.

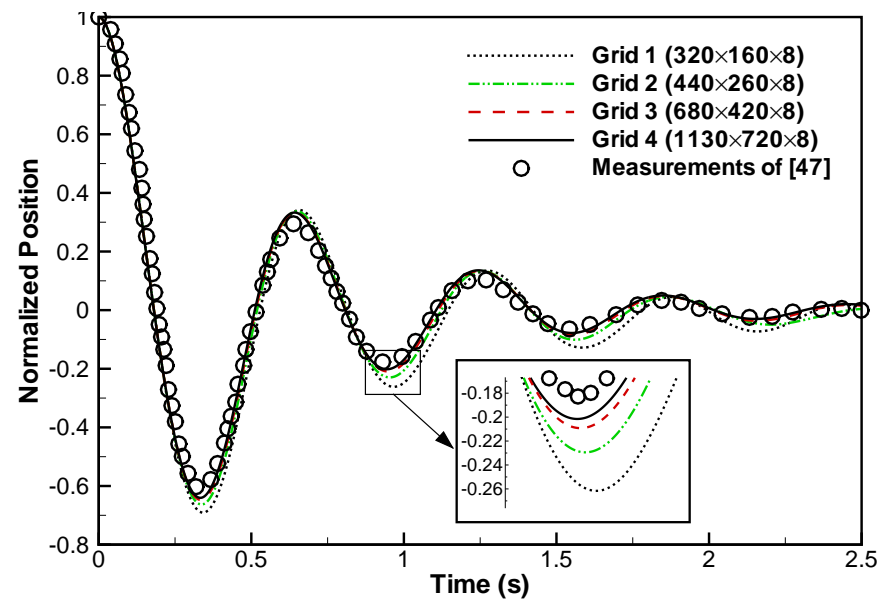

Figure 15: FSI simulation of heave decay of a circular cylinder. Normalized position of the cylinder for several grid refinements (grids 1 to 4 ) and the experimental data of Ito [47]. The time step size employed is $0.0005 s$.

free surface thin layers of positive and negative vorticity form along the interface. A system of waves is generated and starts propagating away from the cylinder in the horizontal direction. The so induced curvature of the free surface and the associated pressure gradients that develop in the flow give rise to a complex vorticity field as evident in figure 13d-h. A distinct characteristic of the flow on the air side is the formation of dipole vortical structures that initially rise away from the surface but then alter their propagation direction to curve downward toward the surface. This complex flow patterns in the air flow, which could only be predicted in grid 4, are the result of flow separation off the crest of the waves induced by the motion of the cylinder (see figure 13d). Even though experimental measurements to allow us to assess the accuracy of these findings are not available for this case, it is important to note that similar vortex dipole structures have also been observed in a recent numerical study by Iafrati et al. [4] who simulated the modulation instability process of water waves. 
To analyze the accuracy of the FSI algorithm and demonstrate the accuracy of the proposed PPBC method for calculating the force we compare in figures 14 and 15 the measured ([47]) and computed normalized position of the cylinder as function of time. In figure 14 we compare the accuracy of the PPBC method with that of the standard method for calculating the force on the body by carrying out FSI simulations with both methods on grid 3 . It is seen in this figure that the PPBC method is able to accurately predict the frequency as well as the amplitude of oscillation. On the other hand, when the standard method is implemented the resulting position of the cylinder is shifted upwards and does not agree well with the measurements. This finding is consistent with the previously discussed (see section 3.3) limitation of the standard method, which over predicts the net force acting on the structure. To quantitatively compare the accuracy of the two methods, the temporally averaged error of the position of the cylinder is calculated using the following expression:

$$
E=\frac{1}{n} \sqrt{\sum_{i=1}^{n}\left(z\left(t_{i}\right)-z_{\exp }\left(t_{i}\right)\right)^{2}}
$$

where $z$ is the vertical position of the center of the cylinder with respect to the calm free surface, the subindex exp indicates the experimental results ([47]), and $n$ is the total number of time steps. The error for the PPBC method is $E=6.13 \cdot 10^{-4}$ while for the standard method nearly one order of magnitude higher $E=1.28 \cdot 10^{-3}$. Therefore, this test case as well as the convergence studies we reported in the previous section of this paper establish clearly the superiority of the PPBC method for calculating the pressure force acting on floating structures. For that this method is used in all subsequently reported test cases.

Finally, in figure 15 we report the results of a grid sensitivity study using the PPBC method in grids 1-4. It is evident that as the grid is refined the solution converges monotonically toward the experimental measurements, thus, establishing the accuracy of our coupled FSI formulation. One small discrepancy between simulations and experiments is that the latter appear to exhibit a slightly higher damping factor, which may be explained by the friction present in the experimental apparatus which is not taken into account in the simulations. Note that the damping coefficient $C$ in equations (9) is set to zero. Overall, however, the computed results are in excellent agreement with the experiments.

\subsection{FSI case 2: Free roll decay of a rectangular barge}

In this case we seek to validate the FSI algorithm under a single degree of freedom in the rotational direction by applying it to simulate a rectangular barge like structure identical to that studied experimentally by Jung et al. [48]. The barge is $0.9 m$ long, $0.3 m$ wide and $0.2 m$ tall, and it is positioned in a $35 m$ long rectangular channel with a $0.9 m$ wide section and water depth of $0.9 \mathrm{~m}$. The barge is allowed to rotate freely with respect to its center of gravity (CG), which coincides with the calm free surface water level, and its rotational inertia is $I=0.236 \mathrm{kgm}^{2}$. It is initially inclined at an angle of 15 degree with respect to the free surface level and its draft is $0.05 \mathrm{~m}$. The configuration is illustrated in figure 16.

Similar to the heave cylinder case presented in the previous section, this is a 3D simulation where the barge width 


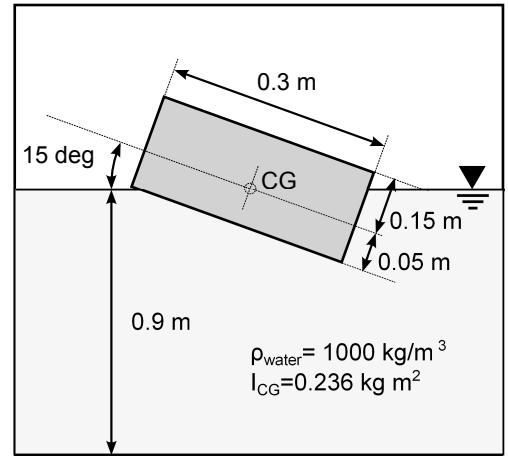

Figure 16: FSI simulation of roll decay of a rectangular barge. Schematic description of the barge configuration studied experimentally by Jung et al. [48].

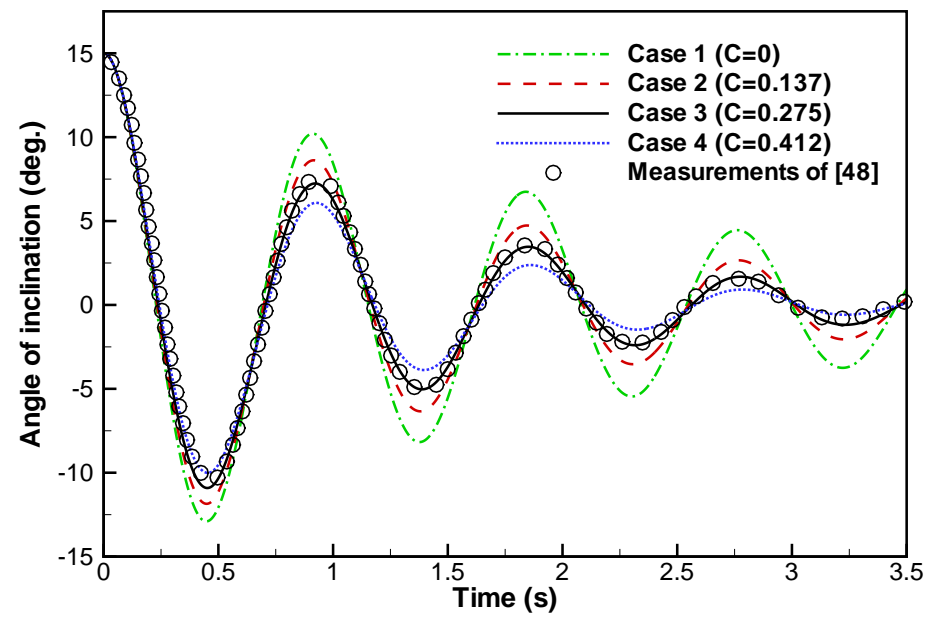

Figure 17: FSI simulation of roll decay of a rectangular barge. Angle of inclination of the barge for the several cases of artificial damping, and the experimental data of Jung et al. [48]. The results have been obtained on a grid with uniform near-body spacing of $0.001 \mathrm{~m}$ and a time step of $0.0005 \mathrm{~s}$.

coincides with the width of the channel. Free-slip conditions are applied at the side walls of the channel and the Reynolds number based on the barge width, and the maximum value of velocity achieved by its corner is equal to $R e=45,000$. A two-region grid structure is employed for this case as for previous cases. In the inner region centered around the structure $([-0.8,0.8]$ in the horizontal direction and $[-0.3,0.3]$ in the vertical direction) the grid is uniform with grid spacing both in the horizontal and vertical directions equal to $0.001 \mathrm{~m}$. Outside of this inner region the grid is gradually coarsened away from the structure using the hyperbolic stretching function. The overall grid size we employ herein consist of approximately 10.5 million, with $1300 \times 740 \times 11$ nodes in the horizontal, vertical, and spanwise directions, respectively. As for the previous case, we carry out 3D LES but without resolving the spanwise direction, essentially assuming that the underlying flow phenomena that govern the dynamics of the structure response are 2D. The time step for the simulation is $0.0005 s$ and the LC-FSI algorithm is used. 


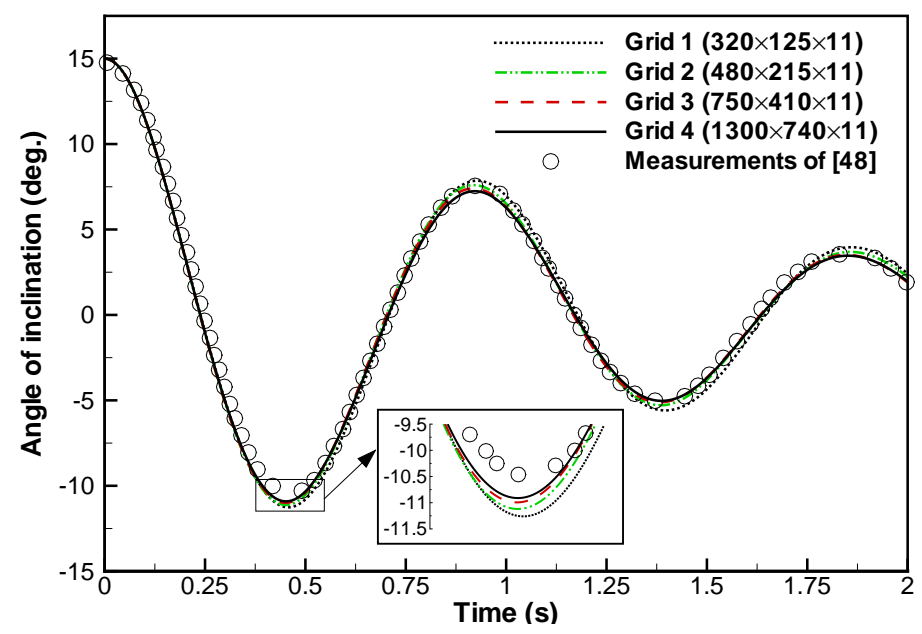

Figure 18: FSI simulation of roll decay of a rectangular barge. Computed angle of inclination of the barge on a series of refined grids (grids 1 to 4 ) and for fixed value of $\mathrm{C}=0.275$, and the experimental data of Jung et al. [48]. The grid spacing near the barge for each of the four grids is $0.008 \mathrm{~m}$, $0.004 m, 0.002 m$ and $0.001 m$, respectively. The time step size employed is $0.0005 \mathrm{~s}$ and the interface thickness $\epsilon$ is $0.02 m$

The damping of the roll motion of the barge can in general be due to the combined action of viscous dissipation of the energy transferred from the barge to the flow and the friction of the experimental apparatus [48]. Our method can in principle resolve the former effects since it can capture the complex vortical field that emerges as the barge oscillates, including vortex-barge and vortex-vortex interactions (see figure 19), which are responsible for dissipating the energy transferred from the barge to the flow. However, the apparatus-specific frictional damping is not know a priori and needs to be incorporated in the simulation by specifying the damping coefficient $C$ in the equation of motion (10). While in the previous case we found that friction effects were small and a value of $\mathrm{C}=0$ was adequate for obtaining accurate solutions, in the present case the computed amplitude of oscillations is found to be sensitive to the value of $\mathrm{C}$. To investigate this sensitivity, we consider four test cases with increasing value of damping coefficient: $C=0, C=0.1375, C=0.275$, and $C=0.425$ for cases 1 to 4 , respectively. The angular position of the barge as a function of time calculated for each of the four cases using the same computational grid for all cases is compared with the experimental results of [48] in figure 17. It is evident from this figure that the numerical model can resolve the experimentally documented natural frequency of the structure with excellent accuracy regardless of the value of C. The value of friction coefficient, however, does affect the amplitude of the oscillation. For example, for the $\mathrm{C}=0$ case the amplitude is over-predicted and as $\mathrm{C}$ is increased the amplitude, as one would anticipate, is systematically dampened. The best fit with the experimental data is clearly obtained for Case 3. This test case underscores the difficulties involved in using such a case to validate a coupled FSI code since the friction of the experimental apparatus is shown to affect the accuracy of the results. Nevertheless, the results in figure 17 clearly show that the frequency of the oscillation does not depend on $\mathrm{C}$ and is resolved with good accuracy for all four cases. Therefore, the results presented herein can be best viewed as a calibration of the friction coefficient in our coupled FSI model for this 


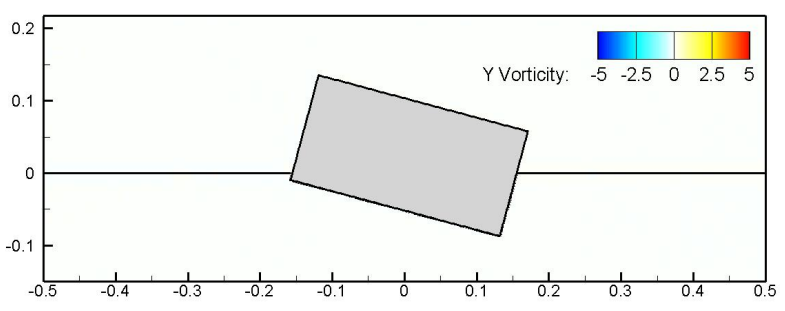

(a) $\mathrm{t}=0.0 \mathrm{~s}$

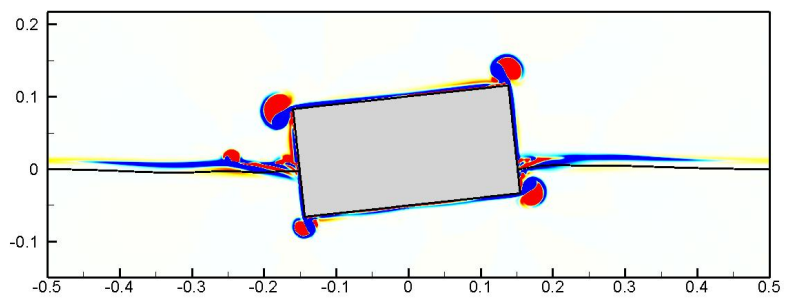

(c) $t=0.6 \mathrm{~s}$

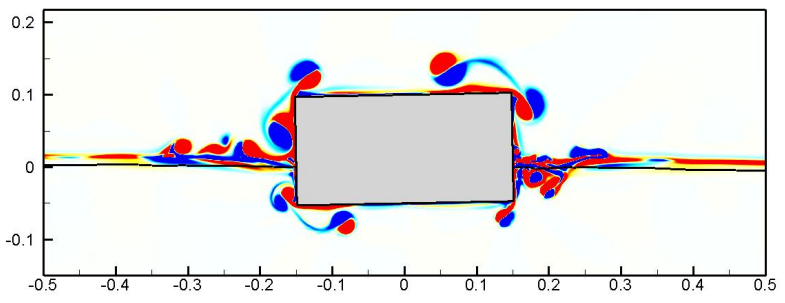

(e) $\mathrm{t}=1.2 \mathrm{~s}$

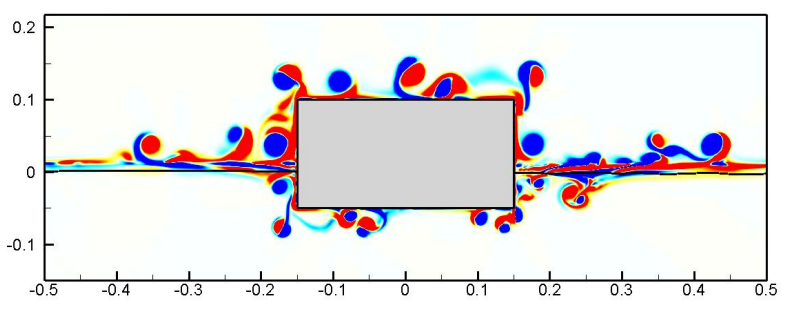

(g) $\mathrm{t}=2.1 \mathrm{~s}$

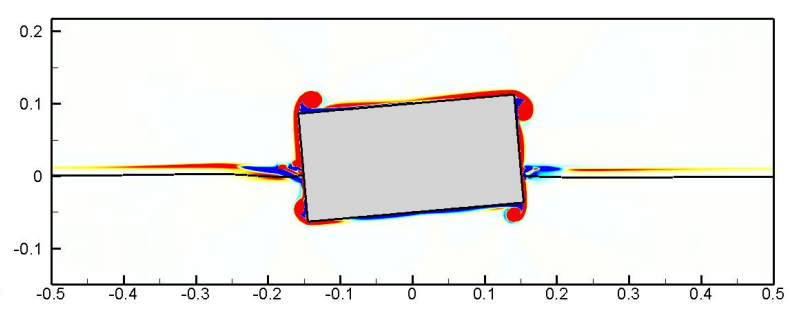

(b) $t=0.3 \mathrm{~s}$

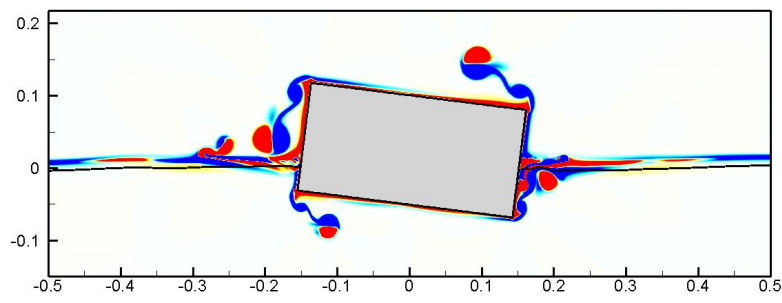

(d) $t=0.9 \mathrm{~s}$

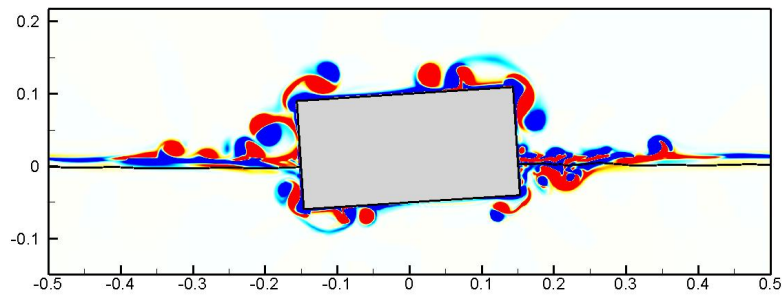

(f) $\mathrm{t}=1.4 \mathrm{~s}$

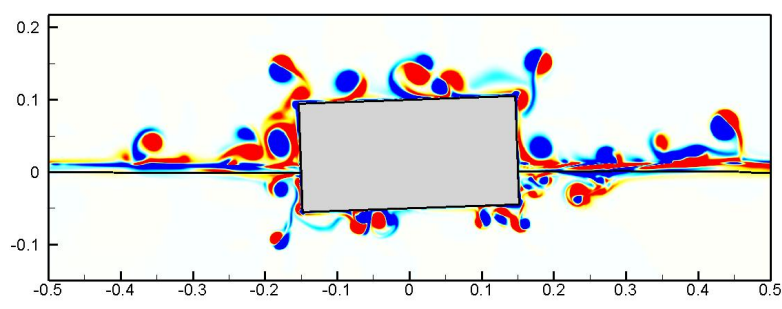

(h) $t=2.4 \mathrm{~s}$

Figure 19: FSI simulation of roll decay of a rectangular barge. Several snapshots of the calculated position of the barge, the free surface, and corresponding out-of-plane vorticity contours are shown in these figures. The solution has been obtained on a grid with near-body spacing equal to $0.001 \mathrm{~m}$, the time step size is $0.0005 \mathrm{~s}$, the interface thickness $\epsilon$ is $0.02 \mathrm{~m}$, and the damping coefficient $C$ is 0.275 .

case under the assumption that our solver is able to accurately resolve viscous effects due to the flow. To explore the validity of this assumption, and since the viscous damping depends on the ability of the computational grid to resolve the underlying flow structures, we carry out another sensitivity study using the value of $\mathrm{C}$ that yielded the best agreement with the experimental data in figure $17(\mathrm{C}=0.275)$ but systematically refining the computational grid. The results of this study are summarized in figure 18, which compares the computed amplitude on the four successively 
finer grids with the same value of $\mathrm{C}$ and the same computational time step and for the first 2 secs of the decay process. It is evident from this figure that the calculated dynamic response of the barge is essentially the same on all four grids. Only minor differences are observed between the coarser grids 1 and 2 and the two finer grids 3 and 4 . The solution, however, converges monotonically as the grid is refined.

The flow patterns that develop in the air and water phases during the various phases of the barge oscillation are illustrated for $C=0.275$ in figure 19, which shows instantaneous contours of spanwise vorticity along with the corresponding shape of the free surface. As seen in this figure, the flow at the early stages of the oscillation is dominated by vortex dipoles shed from the four corners of the barge align with thin layers of vortices generated along the undulating free surface. The mechanism for generating free surface vorticity appears similar to that we discussed in the previous cylinder case and is associated with the unsteady system of wave induced by the motion of the structure. As the oscillation advances, the corner-shed dipoles are seen to, depending on the specific corner of the barge off which they were shed, impinge either on the free surface or the flat surface of the barge giving rise to a very complex vortical flow. Another mechanism for enhancing the complexity of the flow is observed at the later stages of the oscillation $(t>0.9 s)$ when the barge-induced waves propagate away from the structure and give rise to vortex dipoles that are shed off the free surface and rise into the air phase. This is clearly seen in figure 19h, which shows a series of three consecutive dipoles propagating away from the structure and rising into the air.

In summary, the results we have presented herein demonstrate the ability of the coupled FSI, level set formulation to simulate rotational degrees of freedom and resolve the very complex flow phenomena that arise as a result of the coupled interaction between the floating structure and the free surface. Our simulations along with the results we presented above for the cylinder case and recent numerical simulations of Iaffratti et al. [4] further show that the formation of vortex dipoles in the air-phase induced by waves propagating along the free surface appears to be a ubiquitous feature of such flows. Our results suggest that such phenomena occur regardless of how the waves have been generated and clearly point to the need for further studies to probe their underlying physics.

\subsection{FSI case 3: Free falling wedge}

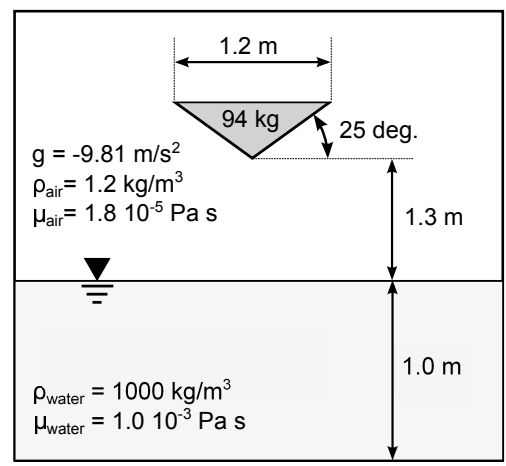

Figure 20: Schematic description of the free falling wedge configuration studied experimentally by Yettou et al. [49]. 


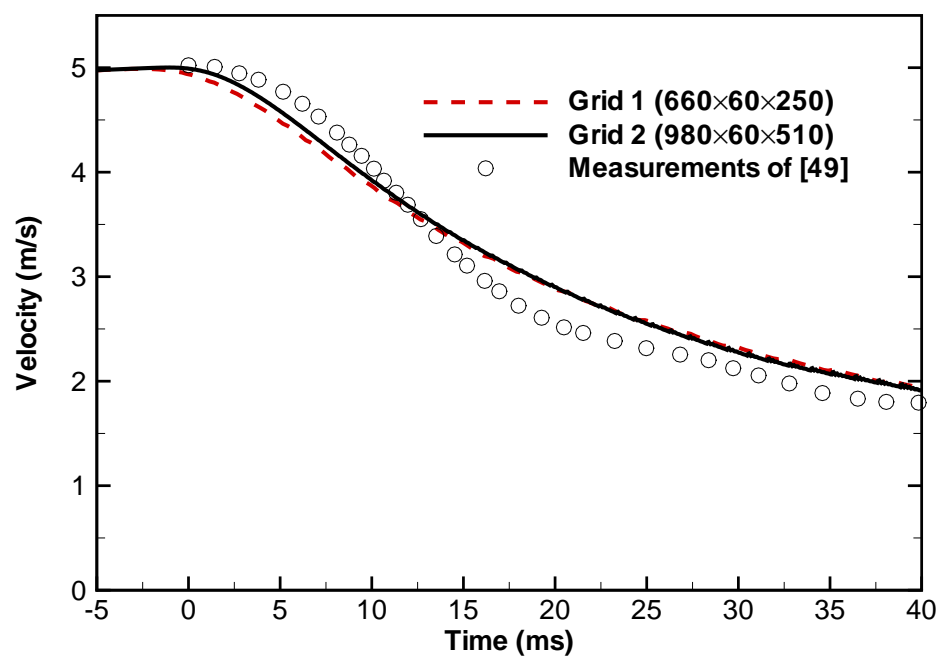

Figure 21: FSI simulation of a free falling wedge. Velocity of the wedge at the initial stage of the impact computed with the PPBC method on grids 1 and 2, and the experimental data of Yettou et al. [49].

The last test case we consider herein involves the simulation of a geometrically complex body, a 3D wedge, falling freely and impinging with its pointed keel into the free surface. The geometrical complexity of the structure along with the large pressure and velocity gradients that develop as the structure's keel impinges on the surface make this the most challenging of all the cases we have studied in this paper. The specific test case we simulate corresponds to that studied experimentally by Yettou et al. [49], who reported detailed data sets of the wedge velocity and position as function of time. In this work we focus on both the dynamics of the wedge motion and the analysis of the coupled air and water complex flow patterns, which have not been addressed in previous studies. For the most part, previous works on wedge impact problems have used laboratory experiments $[50,51,49,52]$ and computational models $[53,54,55,50,51,56,1,2]$ to investigate the dynamics of the wedge motion but reported only limited information about the free surface and flow patterns.

The simulated 3D body has a symmetric wedge-shaped section with a 25 degree dead-rise angle. Its mass is $94 \mathrm{Kg}$, which is equivalent to a structural density of $\rho=466.6 \mathrm{Kg} / \mathrm{m}^{3}$. Initially, the keel of the wedge is placed $1.3 \mathrm{~m}$ above the free surface and starts moving downwards under the action of gravity towards the free surface. The geometrical configuration of the simulation is illustrated in figure 20. The flow Reynolds number based on the wedge length and its maximum velocity is of the order of $R e=5 \cdot 10^{5}$.

In the experiment of [49], the length of the wedge in the spanwise direction is $L=1.2 \mathrm{~m}$, while the channel width is $2 m$, i.e. there is a gap of $0.4 m$ between the wedge and the lateral walls of the channel. In our simulation these gaps are taken into account and resolved by the spanwise grid, which consists of 60 nodes with 12 nodes resolving each gap. The lateral boundaries of the computational domain as well as the wedge surface are treated as no-slip walls.

The computational domain is a $30 \mathrm{~m}$ long channel, $2 \mathrm{~m}$ wide with and a water depth of $1 \mathrm{~m}$, and the wedge is located at the center section of the channel. Two grids of different resolution have been used, both of which exhibit a similar 


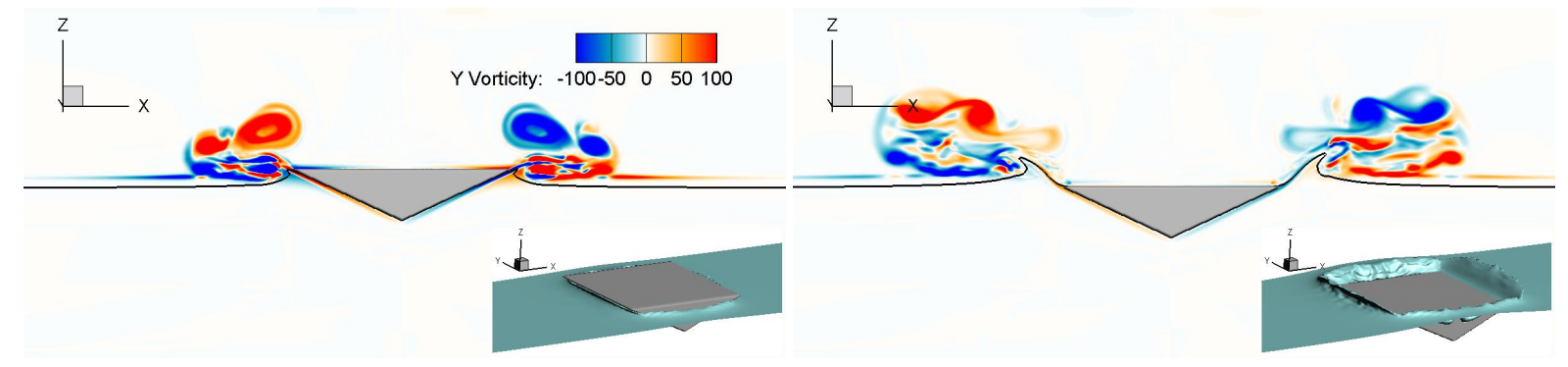

(a) $t=0.1 \mathrm{~s}$

(b) $t=0.2 \mathrm{~s}$

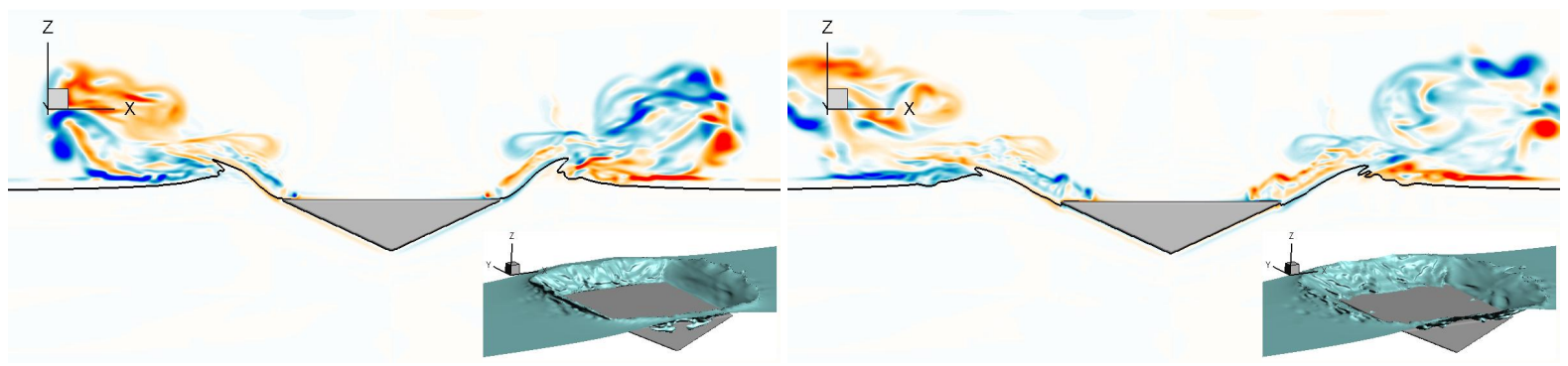

(c) $t=0.3 \mathrm{~s}$

(d) $t=0.4 \mathrm{~s}$

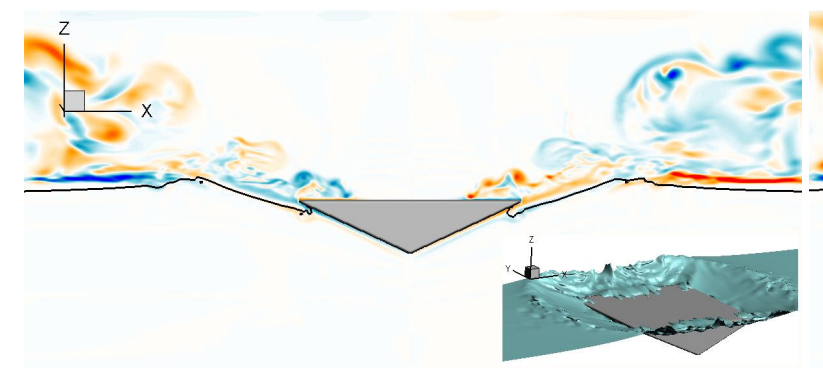

(e) $t=0.5 \mathrm{~s}$

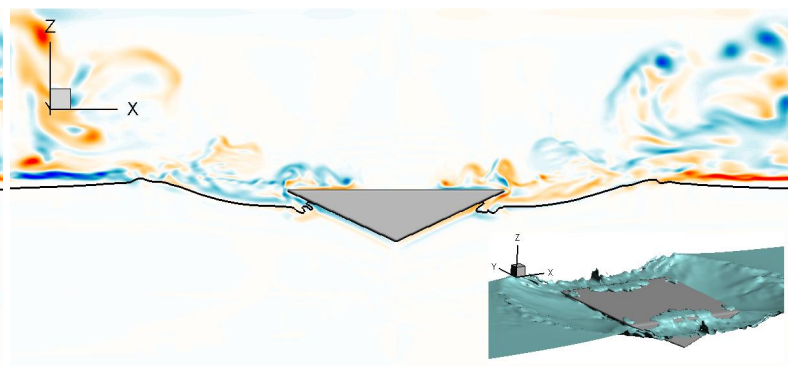

(f) $t=0.6 \mathrm{~s}$

Figure 22: FSI simulation of a free falling wedge. Several snapshots of the calculated position of the wedge, the free surface, and corresponding out-of-plane vorticity contours are shown in these figures at the cross middle plane $(Y=0)$. A small 3D view of the wedge is superposed. The solution has been obtained on grid 1 , which has near-body spacing equal to $\Delta x=\Delta z=0.005 L$, and a time step of $0.00025 \mathrm{~s}$ has been used.

inner/outer region structure we employed in the previous simulations. In grid 1 the constant grid spacing near the structure is of $0.005 \mathrm{~L}$ both in the longitudinal and vertical directions while in grid 2 the corresponding grid spacing is of $0.0025 \mathrm{~L}$. Both meshes have 60 grid nodes in the span-wise direction and the overall grid size is approximately 10 and 30 million, respectively.

The dimensions in the horinzontal (x), spanwise (y) and vertical (z) directions for grid 1 are $660 \times 60 \times 250$ and for grid $2980 \times 60 \times 510$. The inner rectangular region of constant grid spacing that encloses the wedge is of size [-0.6, $0.6]$ in the horizontal direction, and $[-0.6,0.4]$ in the vertical direction. In the outer region, the stretching ratio in the $\mathrm{x}$ direction has been kept reduced $(1.001)$ and constant along the following interval $(0.6<|x|<2.5)$ which coincides with the area of high vorticity generated by the wedge. Away from this interval the stretching ratio is increased 


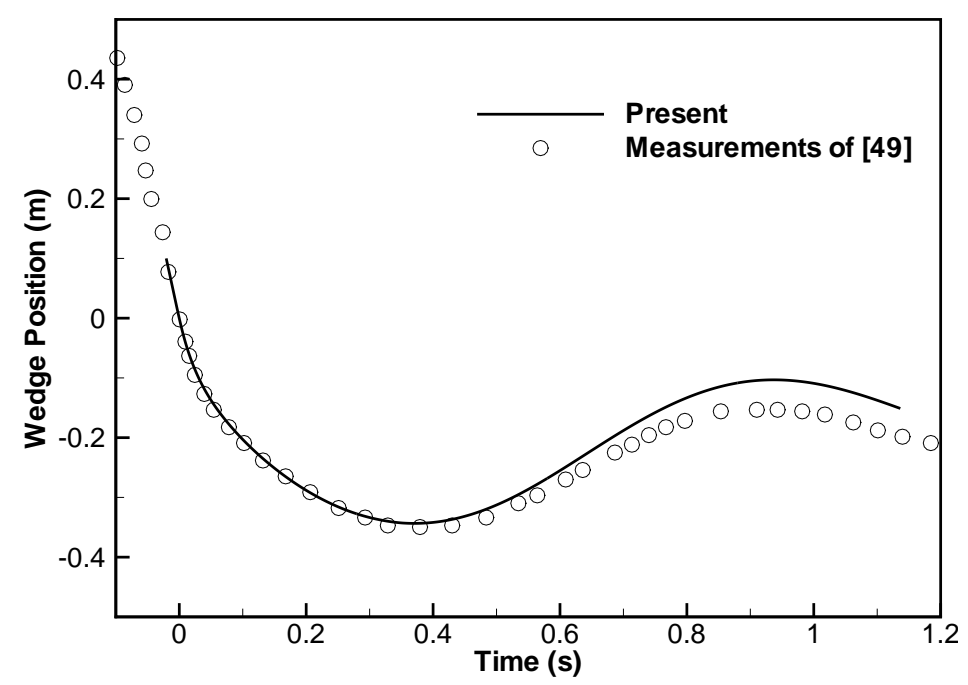

Figure 23: FSI simulation of a free falling wedge. Vertical position of the wedge computed with the PPBC method on grid 1, and the experimental data of Yettou et al. [49].

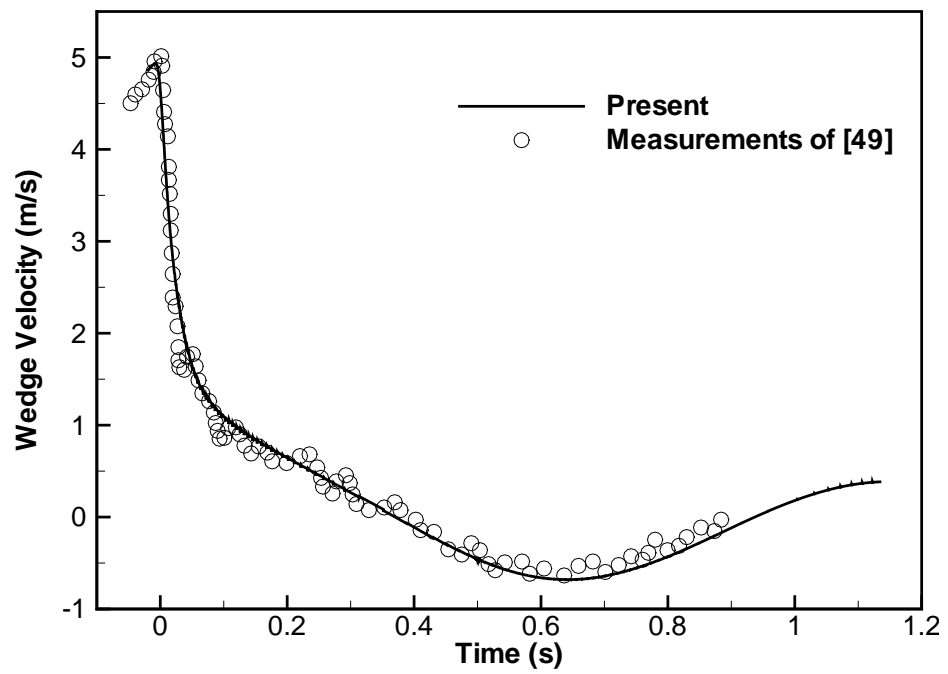

Figure 24: FSI simulation of a free falling wedge. Vertical velocity of the wedge computed with the PPBC method on grid 1, and the experimental data of Yettou et al. [49].

progressively up to a limit of 1.05 .

Due to the large forces that develop on the structure during impact, this test case was proven especially challenging to simulate for the FSI algorithm. Using loose coupling FSI did not yield converged solutions on any of the computational grids and regardless of how small time step we selected. During these initial tests with the loose coupling algorithm, the FSI scheme was consistently found to become unstable approximately between $0.1 s$ and $0.2 s$ from the start of the simulation. Converged solutions were obtained only when the strong coupling FSI algorithm was employed and with reduced time steps equal to $\Delta t=0.00025 \mathrm{~s}$ and $\Delta t=0.00005 \mathrm{~s}$ for grids 1 and 2 , respectively. 
Moreover the use of the Aitken acceleration technique was found essential for increasing the efficiency of the stringcoupling FS algorithm, reducing the number of SC-FSI sub-iterations required for achieving convergence by a factor of 2-3: from 10-12 without Aitken to only 4-5 with Aitken.

Another important issue for enhancing the robustness and accuracy of the algorithm in this case was the need to use an interface thickness $\epsilon$ equivalent to 8 grid cell spacings, and a reduced reinitialization time step of $\Delta \tau=0.02 \Delta x$. The overall re-initialization time $\tau$ was taken to be approximately $10 \%$ of the time required for a full reinitialization. As shown before, this value is adequate for obtaining accurate solutions.

The most challenging, from the computational standpoint, stage of this problem is the first $40 \mathrm{~ms}$ of the process during which the wedge impinges on the free surface and decelerates rapidly from $5 \mathrm{~m} / \mathrm{s}$ to $2 \mathrm{~m} / \mathrm{s}$. During this initial stage we carry out simulations on both the coarse and fine meshes and the calculated temporal variation of the the wedge velocity is compared with the experiments of [49] in figure 21. It is evident from this figure that the computed results on both grids are in good agreement with each other and in reasonable overall agreement with the experimental measurements.

To demonstrate the complex flow patterns that develop as the wedge impinges on the free surface, we show in figure 22 a series of snapshots of the simulated free surface patterns along with contours of the $Y$ component of the vorticity on the $Y=0$ plane. As seen in this figure, as the wedge impinges on the surface it generates a wave on each of its sides. These waves steepen as they propagate away from the wedge and ultimately break leading to massive separation off the the wave cusp and production of large-scale vortical structures of opposite sign in the air phase. This breaking wave generated vorticity is entirely confined in the air phase and ultimately breaks up into smaller scales giving rise to a highly turbulent flow state that rises for several wedge heights above the free surface. These results are entirely consistent with the already discussed findings of Iafrati et al. [4] who showed that in breaking wave phenomena most of the energy is transferred from the wave to the air phase. [4] also showed that vortical structured generated in the air phase near the free surface during wave breaking are able to penetrate into the air phase at significant heights above the surface, a phenomenon which is also observed in our simulations. We note, however, that due to the impact of the wedge on the surface, the condition we have simulated herein is more severe than previously studied cases with enormous amounts of energy transferred from the wedge, to the breaking waves and ultimately to the air phase.

In figures 23 and 24, we compare the simulated temporal variation of the wedge position and velocity over a longer time interval-namely, the first $1.2 \mathrm{~s}$ of the process-with the experimental measurements of [49]. The simulation results shown in these figures have been obtained on grid 1 since as shown in figure 21 this grid is adequate for accurate predictions of the structure response. It is readily seen from figures 23 and 24 that the computed results are in very good agreement with the measurements, capturing both the frequency and amplitude of the wedge oscillation with good accuracy. These results further reinforce the accuracy of the PPBC method we developed herein for calculating the pressure force acting on floating structures. However, as the simulation advances in time, it is observed an increasing discrepancy in the prediction of the wedge oscillation amplitude (between $0.7 s$ and $1.1 s$ ). A reason 
can be extracted by looking at the insets in figure 22 that show the 3D structure of the zero level set to illustrate the complex phenomena that are simulated during wedge impingement and wave breaking. As seen in these snapshots, when the wedge approaches maximum depth water over topping is observed. During this process a significant amount of water is trapped on top of the structure as it starts moving upward. As the layer of trapped water spreads laterally to occupy a larger area its thickness is reduced. The level set approach we employ herein is an inherently diffused interface method and as such free surface phenomena can be captured up to scales for which the resolution of the grid is sufficiently high to resolve the air-water interface. When the water thickness becomes unresolvable, consecutive interfaces tend to merge meaning that the water mass balance is not fully conserved. Ultimately this under-resolved layer of over-topped water will spuriously disappear reducing the gravity force acting on the wedge. This issue could, at least in part, be responsible for the slight over-prediction of the vibration amplitude observed in figure 23 at times greater than $0.8 s$ when the wedge is moving upward. The only practical approach to correctly resolve this problem is to employ adaptive mesh refinement, which will enable local refinement of the mesh at resolution sufficiently fine for resolving the interface thickness of over-topped water. This is beyond the scope of the present work but will be pursued in future work. Also, another challenge for accurately resolving over-topping phenomena stems from the need to take into account the surface tension force, which will undoubtedly become important in determining the dynamics of thin pockets of water trapped in the air phase on top of a solid structure. Our level set approach can incorporate the surface tension force (see eqn 2) but this needs to be done in conjunction with sufficient grid resolution to resolve local variations of the interface at scales for which surface tension forces become important. This is also beyond the scope of our work. Nevertheless, this resolution limitation notwithstanding, the proposed method is able to simulate the dynamic response of the structure with reasonable accuracy.

Finally, in figure 25 we present several snapshots of the simulated 3D coherent structures visualized with isosurface of q-criterion [57] to illustrate the richness of the ensuing air and water dynamics as the waves off the sides of the wedge steepen and break. As seen, the massive separation zone induced by the breaking waves is dominated by a series vortex loops, arch vortices, and hairpin vortices. It is also evident from sub-figures 22 (a) and (b), corresponding to times $t=0.1 \mathrm{~s}$ and $t=0.2 \mathrm{~s}$, that the 3D complexity of the simulated flow grows rapidly as the waves steepen and begin to break and the flow transitions from laminar to turbulent.

In summary, the results we presented herein show that the proposed coupled FSI level set approach is able to accurately simulate a very challenging case involving large forces on the impinging structure, wave-breaking, and overtopping. The ability of the method to also resolve the complex dynamics of the flow that emerges in the air phase during wave breaking was also illustrated.

\section{Conclusions}

We developed, validated and demonstrated the predictive capabilities of a novel FSI model for simulating arbitrarily complex floating rigid bodies interacting with nonlinear free surface flows. The proposed method integrates 
the FSI-CURVIB method of Borazjani et al. [21] with a level set approach along with a new method for calculating forces due to pressure on submerged structures in two-phase flows.

The so-called pressure projection boundary condition method was shown to mitigate the difficulties encountered when calculating the force on the structure using the standard method developed by Borazjani et al. [21], which employs integration of the pressure on the surface of the volume consisting of all immersed boundary grid cells in the CURVIB method around the structure. While this standard approach works well in single-phase flows, in twophase flow problems with submerged bodies it does not account for the force imparted on the structure due to the large density difference between the air and water enclosed between the immersed boundary surface and the body and gives rise to a first-order accurate calculation of the pressure force. The proposed PPBC approach employs the normal momentum equation to the body to obtain a more accurate representation of the pressure field on the body via a series of successive projection steps. Numerical tests clearly showed that the proposed method not only reduces the error in the calculation of the force by nearly one order of magnitude relative to the standard approach but also yields near second-order accurate convergence rate for the force and results that are in significantly better agreement with experimental measurements. While the PPBC method was developed and demonstrated herein in the context of the CURVIB approach it is general and should be readily applicable to other sharp-interface immersed boundary methodologies.

To demonstrate the accuracy of the coupled FSI, level set implementation for free surface-body interactions we simulated a series of test cases, including forced motion problems and coupled FSI problems. We showed that for all simulated cases the proposed method is able to replicate with good accuracy the structural response of several laboratory experiments including a free decay test of a circular cylinder, a roll decay test of a rectangular structure, and a free falling wedge impacting the free surface.

In order to prevent the formation of instabilities in problems involving complex air-water interface phenomena, the use of a large interface strip thickness $\epsilon$ as well as reduced reinitialization time steps sizes $\Delta \tau$ was found necessary. To reduce the computational cost for implementing these remedies, we systematically investigated the influence of using an overall reinitialization time lower than the time required for full reinitialization of the interface strip. We showed that for the falling cylinder case using a time equivalent to reinitializing $10 \%$ of the interface strip was able to predict in sufficiently high accuracy the formation of the breaking wave formed at the side of the cylinder.

The most challenging case we simulated was that of a wedge impinging on the free surface. Large pressure gradients and forces develop on the structure as it impinges on the surface and decelerates rapidly, which made the solution of the FSI problem especially challenging. Even for this case, however, our method was able to obtain converged solutions but required the use of strong coupling FSI in conjunction with the Aitken method. The latter technique was critical for efficient FSI iterations, since it reduced the number of strong-coiling sub-iterations required for convergence by fifty percent or more.

Our simulations elucidated the rich 3D dynamics resulting in the air phase as the waves induced by the impinging on the free surface wedge break, and showed that most of the energy from the breaking waves is ultimately transferred 
to the air phase. Massive separation off the cusp of the breaking waves gave rise to complex coherent structures dominated by loops, arch, and hairpin vortices forming an intertwined web of vortical structures that ultimately lead to the flow transitioning to turbulence. The resulting turbulent flow in the air phase was found to grow upward and persist at a significant elevation above the free surface. All these findings are in accordance with the recent findings of Iafrati et al. [4] who also showed that flow separation off breaking waves is the key mechanism for producing turbulence in the air phase. Overall the computed results demonstrated the potential of our method as a powerful tool for simulating the coupled interaction of complex floating structures with a free surface.

Our simulations for the wedge case exposed a limitation of the method when structure over-topping occurs. Our method was able to capture this complex phenomenon, which leads to pockets of water entrapped on the flat surface of the wedge in the air phase. As these pockets, however, spread laterally and grow thiner ultimately reach the scale of resolution of the level set method (the thickness of the interface that can be resolved on a given grid) and spuriously disappear. This is an inherent limitation of the level set approach and can only be resolved by local grid refinement, which can be made practical via an adaptive mesh refinement approach. Such algorithmic advances will be pursued as part of our future work.

Finally we note that for all cases we considered herein surface waves were generated as the result of a structure interacting with the free surface. However, to extend the present method to simulate offshore structures, such oil platforms, floating wind turbines, or wave energy converters, interacting with realistic ocean waves it is essential to develop an approach for incorporating a given wave field into the level set simulation. Such undertaking is far from trivial and requires novel algorithmic advances. A coupled FSI level set algorithm that can incorporate real-life ocean waves is currently under development and will be presented in a forthcoming publication.

\section{Acknowledgements}

This work has been supported by the US Department of Energy (DE-EE 0005482) and the University of Minnesota Initiative for Renewable Energy and the Environment. Computational resources were provided by the Minnesota Supercomputing Institute.

\section{References}

[1] K. Kleefsman, G. Fekken, A. Veldman, B. Iwanowski, B. Buchner, A volume-of-fluid based simulation method for wave impact problems, Journal of Computational Physics 206 (2005) 363-393.

[2] S. Tanaka, K. Kashiyama, ALE finite element method for FSI problems with free surface using mesh re-generation method based on background mesh, International Journal of Computational Fluid Dynamics 20 (2006) 229-236.

[3] K. Paik, Simulation of fluid-structure interaction for surface ships with linear/nonlinear deformations, Ph.D. thesis, University of Iowa, 2010.

[4] A. Iafrati, A. Babanin, M. Onorato, Modulational instability, wave breaking, and formation of large-scale dipoles in the atmosphere, Physical Review Letters 110 (2013) 184504.

[5] E. Walhorn, A. Klke, B. Hbner, D. Dinkler, Fluid-structure coupling within a monolithic model involving free surface flows, Computers \& structures 83 (2005) 2100-2111. 
[6] B. Ramaswamy, M. Kawahara, Arbitrary Lagrangian-Eulerianc finite element method for unsteady, convective, incompressible viscous free surface fluid flow, International Journal for Numerical Methods in Fluids 7 (1987) 1053-1075.

[7] W. P. Breugem, B. J. Boersma, Direct numerical simulations of turbulent flow over a permeable wall using a direct and a continuum approach, Physics of fluids 17 (2005) 025103.

[8] C. S. Peskin, Flow patterns around heart valves: A numerical method, Journal of Computational Physics 10 (1972) $252-271$.

[9] F. Sotiropoulos, X. Yang, Immersed boundary methods for simulating fluid-structure interaction, Progress in Aerospace Sciences (2013).

[10] R. Mittal, G. Iaccarino, Immersed boundary methods, Annual Review of Fluid Mechanics 37 (2005) $239-261$.

[11] J. Mohd-Yusof, Combined immersed-boundary/B-spline methods for simulations of flow in complex geometries, Annual Research Briefs. NASA Ames Research Center= Stanford University Center of Turbulence Research: Stanford (1997) 317-327.

[12] D. K. Clarke, H. A. Hassan, M. D. Salas, Euler calculations for multielement airfoils using cartesian grids, AIAA Journal 24 (1986) $353-358$.

[13] A. Gilmanov, F. Sotiropoulos, A hybrid cartesian/immersed boundary method for simulating flows with 3D, geometrically complex, moving bodies, Journal of Computational Physics 207 (2005) 457-492.

[14] A. Gilmanov, S. Acharya, A hybrid immersed boundary and material point method for simulating 3D fluid-structure interaction problems, International Journal for Numerical Methods in Fluids 56 (2008) 2151-2177.

[15] A. Gilmanov, S. Acharya, T. Gilmanov, Flow-structure interaction simulations for ballutes in supersonic flow, AIAA Paper 2906 (2009).

[16] S. Haeri, J. S. Shrimpton, On the application of immersed boundary, fictitious domain and body-conformal mesh methods to many particle multiphase flows, International Journal of Multiphase Flow 40 (2012) 3855.

[17] E. Balaras, Modeling complex boundaries using an external force field on fixed cartesian grids in large-eddy simulations, Computers \& Fluids 33 (2004) 375-404.

[18] M.-C. Lai, C. S. Peskin, An immersed boundary method with formal second-order accuracy and reduced numerical viscosity, Journal of Computational Physics 160 (2000) 705-719.

[19] L. Shen, E.-S. Chan, P. Lin, Calculation of hydrodynamic forces acting on a submerged moving object using immersed boundary method, Computers \& Fluids 38 (2009) 691-702.

[20] J. Sanders, J. E. Dolbow, P. J. Mucha, T. A. Laursen, A new method for simulating rigid body motion in incompressible two-phase flow, International Journal for Numerical Methods in Fluids 67 (2010) 713-732.

[21] I. Borazjani, L. Ge, F. Sotiropoulos, Curvilinear immersed boundary method for simulating fluid structure interaction with complex 3D rigid bodies, Journal of Computational Physics 227 (2008) 7587-7620.

[22] I. Borazjani, Numerical Simulations of Fluid-Structure Interaction Problems in Biological Flows, Ph.D. thesis, University of Minnesota, 2008.

[23] L. Shen, E.-S. Chan, Numerical simulation of fluid-structure interaction using a combined volume of fluid and immersed boundary method, Ocean Engineering 35 (2008) 939-952.

[24] J. Yang, F. Stern, Sharp interface immersed-boundary/level-set method for wave-body interactions, Journal of Computational Physics 228 (2009) 6590-6616.

[25] S. Kang, A. Lightbody, C. Hill, F. Sotiropoulos, High-resolution numerical simulation of turbulence in natural waterways, Advances in Water Resources 34 (2011) 98-113.

[26] M. Germano, U. Piomelli, P. Moin, W. H. Cabot, A dynamic subgrid-scale eddy viscosity model, Physics of Fluids A: Fluid Dynamics 3 (1991) 1760-1765.

[27] S. J. Osher, R. P. Fedkiw, Level Set Methods and Dynamic Implicit Surfaces, 2003 ed., Springer, 2002.

[28] A. Iafrati, E. F. Campana, Free-surface fluctuations behind microbreakers: space $\phi$ time behaviour and subsurface flow field, Journal of Fluid Mechanics 529 (2005) 311-347.

[29] A. Iafrati, Numerical study of the effects of the breaking intensity on wave breaking flows, Journal of Fluid Mechanics 622 (2009) 371.

[30] S. Osher, J. A. Sethian, Fronts propagating with curvature-dependent speed: algorithms based on hamilton-jacobi formulations, J. Comput. Phys. 79 (1988) 12-49. 
[31] M. Sussman, E. Fatemi, An efficient, interface-preserving level set redistancing algorithm and its application to interfacial incompressible fluid flow, SIAM J. Sci. Comput. 20 (1999) 1165-1191.

[32] S. Kang, F. Sotiropoulos, Numerical modeling of 3D turbulent free surface flow in natural waterways, Advances in Water Resources 40 (2012) $23-36$

[33] L. Ge, F. Sotiropoulos, A numerical method for solving the 3D unsteady incompressible NavierStokes equations in curvilinear domains with complex immersed boundaries, Journal of Computational Physics 225 (2007) 1782-1809.

[34] G.-S. Jiang, C.-W. Shu, Efficient implementation of weighted ENO schemes, Journal of Computational Physics 126 (1996) $202-228$.

[35] S. Armfield, R. Street, An analysis and comparison of the time accuracy of fractional-step methods for the NavierStokes equations on staggered grids, International Journal for Numerical Methods in Fluids 38 (2002) 255-282.

[36] M. N. Huxley, Exponential sums and lattice points III, Proceedings of the London Mathematical Society 87 (2003) 591-609.

[37] A. Ivic, E. Krtzel, M. Khleitner, W. G. Nowak, Lattice points in large regions and related arithmetic functions: Recent developments in a very classic topic, arXiv preprint math/0410522 (2004).

[38] J. G. van der Corput, Over roosterpunten in het platte vlak:(de beteekenis van de methoden van Voronoï en Pfeiffer), Ph.D. thesis, P. Noordhoff, 1919.

[39] I. Borazjani, F. Sotiropoulos, Vortex induced vibrations of two cylinders in tandem arrangement in the proximity-wake interference region, Journal of fluid mechanics 621 (2009) 321-364. PMID: 19693281.

[40] W. Cabot, P. Moin, Approximate wall boundary conditions in the large-eddy simulation of high reynolds number flow, Flow, Turbulence and Combustion 63 (2000) 269-291.

[41] M. Wang, P. Moin, Dynamic wall modeling for large-eddy simulation of complex turbulent flows, Physics of Fluids 14 (2002) 2043.

[42] J.-I. Choi, R. C. Oberoi, J. R. Edwards, J. A. Rosati, An immersed boundary method for complex incompressible flows, Journal of Computational Physics 224 (2007) 757-784.

[43] B. M. Irons, R. C. Tuck, A version of the aitken accelerator for computer iteration, International Journal for Numerical Methods in Engineering 1 (1969) 275-277.

[44] P. Lin, A fixed-grid model for simulation of a moving body in free surface flows, Computers \& Fluids 36 (2007) 549-561.

[45] S. O. Unverdi, G. Tryggvason, A front-tracking method for viscous, incompressible, multi-fluid flows, Journal of Computational Physics 100 (1992) 25-37.

[46] M. Greenhow, S. Moyo, Water entry and exit of horizontal circular cylinders, Philosophical Transactions: Mathematical, Physical and Engineering Sciences (1997) 551-563.

[47] S. Ito, Study of the transient heave oscillation of a floating cylinder, Ph.D. thesis, MIT, 1971.

[48] K. H. Jung, K. A. Chang, H. J. Jo, Viscous effect on the roll motion of a rectangular structure, Journal of engineering mechanics 132 (2006) 190-200.

[49] E. M. Yettou, A. Desrochers, Y. Champoux, Experimental study on the water impact of a symmetrical wedge, Fluid dynamics research 38 (2006) 47-66.

[50] L. Xu, A. W. Troesch, R. Peterson, Asymmetric hydrodynamic impact and dynamic response of vessels, Journal of Offshore Mechanics and Arctic Engineering 121 (1999) 83-89.

[51] G. Wu, H. Sun, Y. He, Numerical simulation and experimental study of water entry of a wedge in free fall motion, Journal of Fluids and Structures 19 (2004) 277-289.

[52] S. G. Lewis, D. A. Hudson, S. R. Turnock, D. J. Taunton, Impact of a free-falling wedge with water: synchronized visualization, pressure and acceleration measurements, Fluid Dynamics Research 42 (2010) 035509.

[53] M. Greenhow, Wedge entry into initially calm water, Applied Ocean Research 9 (1987) 214-223.

[54] X. Mei, Y. Liu, D. K. Yue, On the water impact of general two-dimensional sections, Applied Ocean Research 21 (1999) 1-15.

[55] R. Zhao, O. Faltinsen, J. Aarsnes, Water entry of arbitrary two-dimensional sections with and without flow separation, in: 21st symposium on naval hydrodynamics, 1997. 
[56] M. Doring, G. Alessandrini, P. Ferrant, Sph simulations of floating bodies in waves, in: Proceedings of the 19th International Workshop on Water Waves and Floating Bodies, 2004.

873

[57] J. Hunt, A. Wray, P. Moin, Eddies, stream, and convergence zones in turbulent flows, Center For Turbulence Research Report CTR-S88 (1988) 
$\int_{y}^{z}$
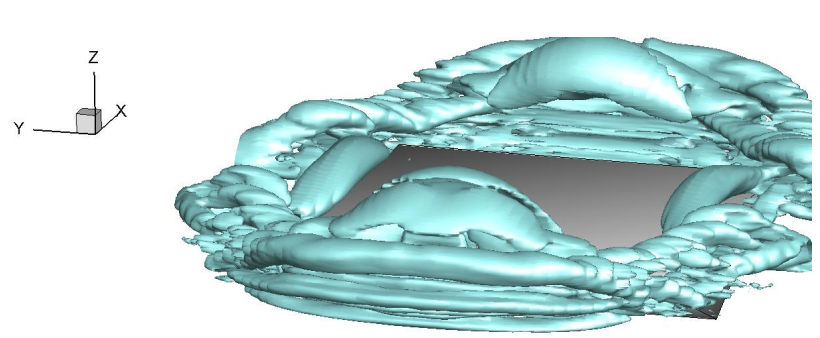

(a) $t=0.1 \mathrm{~s}$

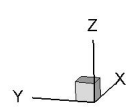<smiles>CC(C)C</smiles>
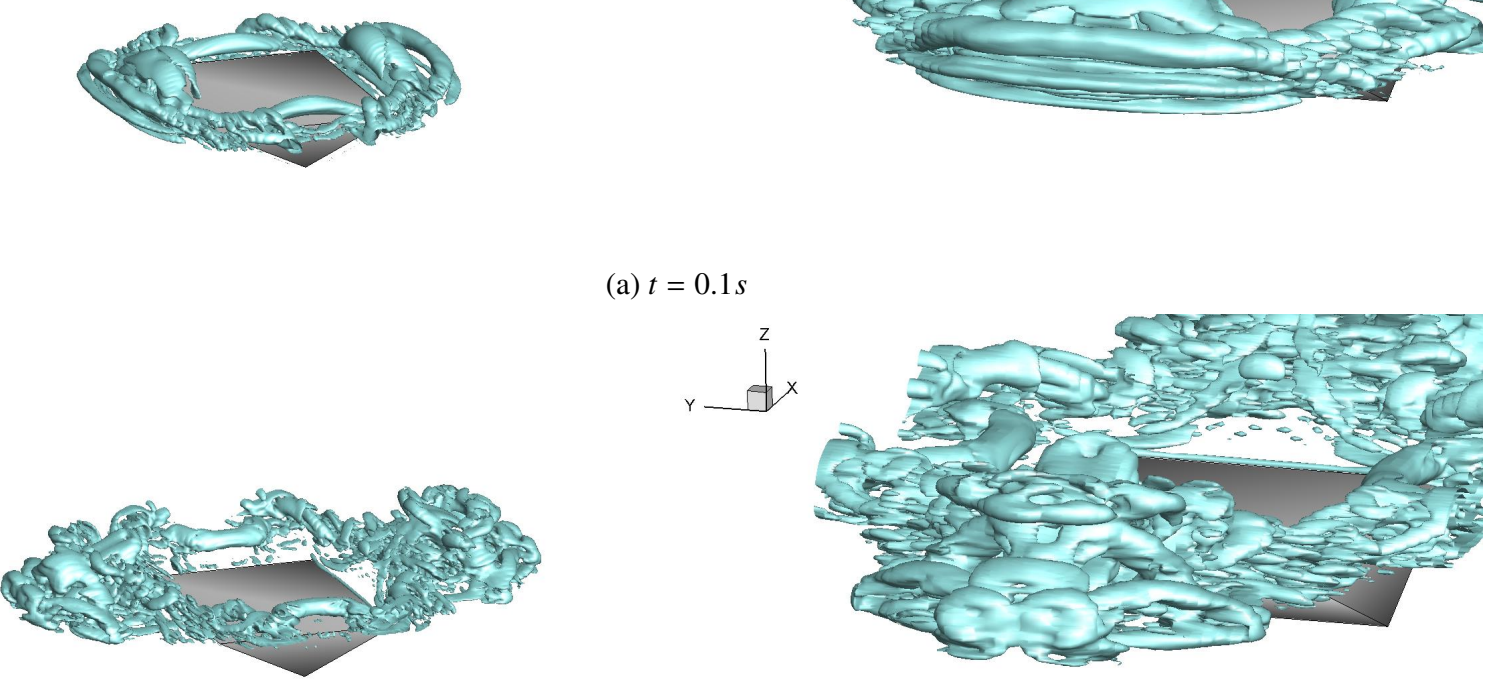

(b) $t=0.2 \mathrm{~s}$<smiles>CP(I)I</smiles><smiles>[V][GaH]</smiles>

(c) $t=0.3 \mathrm{~s}$
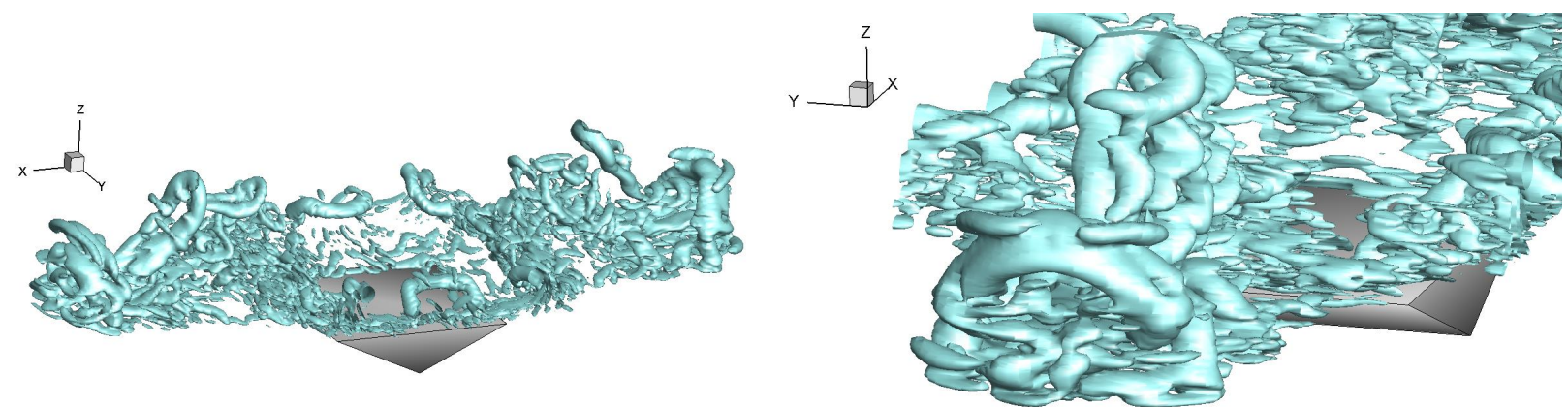

(d) $t=0.4 \mathrm{~s}$

Figure 25: FSI simulation of a free falling wedge. Snapshots showing coherent structures in the flow visualized by the $Q$ criterion $(Q=-200)$. The right figures show a closer view of the coherent structures near the area where wave breaks. The solution has been obtained on grid 1, which has near-body spacing equal to $\Delta x=\Delta z=0.005 L$, and a time step of 0.00025 s has been used. 